\title{
NEW INSIGHT ON GALAXY FORMATION AND EVOLUTION FROM KECK SPECTROSCOPY OF THE HAWAII DEEP FIELDS
}

\author{
LenNoX L. Cowie, ${ }^{1,2}$ AntoinetTe Songaila, ${ }^{1}$ AND Esther M. Hu ${ }^{1,2}$ \\ Institute for Astronomy, University of Hawaii, 2680 Woodlawn Drive, Honolulu, Hawaii 96822 \\ Electronic mail: cowie@ifa.hawaii.edu, acowie@ifa.hawaii.edu, hu@ifa.hawaii.edu \\ J. G. COHEN \\ Palomar Observatory, Mail Stop 105-24, California Institute of Technology, Pasadena, California 91125 \\ Electronic mail: jlc@astro.caltech.edu \\ Received 1996 January 5; revised 1996 May 28
}

\begin{abstract}
We present the results of spectroscopic studies with the LRIS spectrograph on Keck of two of the Hawaii deep survey fields. The 393 objects observed cover a $26.2 \mathrm{arcmin}^{2}$ area and constitute a nearly complete sample down to $K=20, I=23$, and $B=24.5$. The rest-frame $K$-band luminosity function and its evolution with redshift are described. Comparisons are made with other optically selected $(B$ and $I)$ samples in the literature, and the corresponding rest-frame $B$-band luminosity function evolution is presented. The $B$-band counts at $B \sim 24$ are shown to be a mixture of normal galaxies at modest redshifts and galaxies undergoing rapid star formation, which have a wide range of masses and which are spread over the redshift interval from $z=0.2$ to beyond $z=1.7$. The luminosity functions, number counts, and color distributions at optical and IR wavelengths are discussed in terms of a consistent picture of the star-forming history of the galaxy sample. [O II] emission-line diagnostics or rest-frame ultraviolet-infrared color information are used in combination with rest-frame absolute $K$ magnitudes to construct a "fundamental plane" in which the evolution of the global star-formation rate with redshift can be shown, and we find that the maximum rest-frame $K$ luminosity of galaxies undergoing rapid star formation has been declining smoothly with decreasing redshift from a value near $L_{*}$ at $z>1$. This smooth decrease in the characteristic luminosity of galaxies dominated by star formation can simultaneously account for the high $B$-band galaxy counts at faint magnitudes and the redshift distribution at $z<1$ in both the $B$ - and $K$-selected samples. Finally, the overall $K$-band light density evolution is discussed as a tracer of the baryonic mass in stars and compared with the overall rates of star formation inferred from the rest-frame ultraviolet light density as a function of redshift. (C) 1996 American Astronomical Society.
\end{abstract}

\section{INTRODUCTION}

In order to understand the formation and evolution of galaxies we require very large complete redshift samples to faint magnitudes. Such large samples have recently been obtained in the optical with both blue and red selections $(B$ $<24$ : Glazebrook et al. 1995a; Ellis et al. 1996; $I<22$ : the CFRS Survey-see, e.g., Lilly et al. 1995a). These samples contain several hundred to over one thousand galaxies. However, optically selected samples are severely biased against earlier galaxy types at the higher redshifts and so are rather poorly suited to studying the evolution beyond about $z=1$ where, even in the $I$ band, the $K$ corrections become very large and many of the galaxy types disappear from the selection.

Infrared-selected samples minimize this problem because the $K$ corrections are relatively invariant to galaxy type and

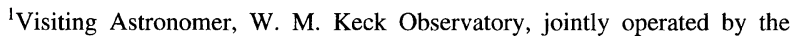
California Institute of Technology and the University of California.

${ }^{2}$ Visiting Astronomer, Canada-France-Hawaii Telescope, operated by the National Research Council of Canada, the Centre National de la Recherche Scientifique of France, and the University of Hawaii. remain small (indeed negative) to high redshift. They are therefore well suited to studying high-redshift populations and are our best method of determining the formation process. Two quite large faint $K(2.2 \mu \mathrm{m})$ or $K^{\prime}(2.1 \mu \mathrm{m})$ samples have recently been published, by Glazebrook $e t$ al. (1995b) and Songaila et al. (1994). However, the former sample extends only to $K=17$, not deep enough to reach the $z \gtrsim 1$ population, and the latter sample, which reaches $K=20$, is somewhat incomplete at the faint end and covers a quite small area at $K \gtrsim 18$.

In this paper we shall describe a much larger, and very faint infrared sample ( $\operatorname{area}=26.2 \mathrm{arcmin}^{2}, K \leqslant 20$ ) of $254 \mathrm{ob}$ jects which we have observed using the LRIS spectrograph (Oke et al. 1995) on the Keck $10 \mathrm{~m}$ telescope. The sample consists of all objects in two $6^{\prime} \times 2^{\prime}$ strips, and is nearly complete to $K=19.5$. To complement the $K$-selected sample we have also observed the $I<23$ or $I<22.5$ and $B<24.5$ objects in the two fields, bringing the total to 393 objects in all.

These three color-selected redshift samples are extremely powerful tools for understanding the evolution of galaxies. We first describe the evolution of the luminosity functions 
and light densities in the various rest-frame colors ( $B$ through $K$ ) out to a redshift of 1.6 and compare the data to the optical and brighter infrared samples in the literature. Next, we use these results to study the evolution of galaxies in a parameter space characterized by the absolute rest $K$ magnitude, $M_{K}$, in combination with either the [O II] or $\mathrm{H} \alpha$ equivalent widths or the rest-frame ultraviolet-infrared color, $U K(A B)$, and the redshift. This is equivalent to a parameterization of each galaxy as a function of mass (shown by $M_{K}$ ) and star-formation rate, and we find that the galaxy population shows a rapid migration in this plane as a function of $z$. At $z>1$, galaxies with near- $L_{*}$ infrared luminosities have [O II] equivalent widths or $U K(A B)$ colors that correspond to very rapid star formation. At lower redshifts, the maximum rest $K$ luminosity of such "forming" galaxies drops smoothly until at the present epoch there are only a few, generally very low luminosity, "forming" galaxies.

We shall refer to this remarkably smooth downward evolution in the maximum luminosity of rapidly star-forming galaxies as "downsizing," since the assembly of the upper end of the galaxy luminosity function is occurring from the top down with decreasing redshift; it is this phenomenon that accounts for the simultaneous rapid evolution in the colors of galaxies and in the normalization of the luminosity function (or equivalently in the $B$-and $K$-band galaxy counts) while leaving the redshift distribution at $z<1$ well characterized by a no-evolution model (Broadhurst et al. 1988; Colless et al. 1990; Steidel et al. 1994). This solution, which relies on the $L_{*}$ galaxies being in place (though still undergoing some star formation at a declining rate) at $z<1$ while sub- $L_{*}$ galaxies are still forming, was originally suggested in Broadhurst et al. (1988) to deal with the $z \sim 0.3$ blue galaxies; however, we now see that the faint $(B \geq 24)$ blue counts contain a variety of galaxies "forming" over a range of redshifts, including massive galaxies at $z>1$. This range of blue galaxies in redshift and luminosity fully accounts, for the faint blue excess in the number counts.

Because the interpretation draws on all the information in all three colors, we have chosen not to divide the paper, which is consequently somewhat long. Many readers may wish to skip the technical details of the observations given in Sec. 2. The reader who is primarily interested in the interpretation may further skip to Sec. 6 , which is intended to be self-contained.

\section{OBSERVATIONS}

The sample was defined as all objects with $K<20$, $I<22.5$ (Kron-Cousins), or $B<24.5$ in two approximately $6^{\prime} \times 2^{\prime}$ areas (exact combined area $=26.2 \operatorname{arcmin}^{2}$ ) surrounding the Hawaii deep survey fields SSA 13 and SSA 22 (Cowie et al. 1994; Songaila et al. 1994) and lying along the E-W direction. The SSA 22 sample was extended to include all $I=23$ objects. The 1950 coordinates for each object (absolute accuracy better than 0.4 ) are given in Tables 1 (a) and 1(b), together with their $K, I$, and $B$ magnitudes and their corresponding numbered identifications on $I$ finding charts of the field, shown in Figs. 1(a) and 1(b). The $K, I$, and $B$ magnitudes are $3^{\prime \prime}$ diameter aperture magnitudes corrected to
$6 "$ diameter with an average offset. The object selection process is described in the previous papers. Tables $1 \mathrm{a}$ and $1 \mathrm{~b}$ are ordered by R.A. and for each field list first the $K<20$ sample, then the additional objects which complete the $I$ sample, and finally those added to complete the $B$ sample are given. A small fraction of the objects are not detected in one of the colors at the limiting $1 \sigma$ magnitudes of $K=21.8, I=25.3$, and $B=26.3$. A negative aperture flux is indicated by a magnitude corresponding to the absolute flux preceded by a negative sign. The SSA 13 catalog contains 174 objects and the SSA 22 catalog 219 objects; the larger number of objects in the SSA 22 field is partly a result of the fainter $I$ selection and partly of the lower galactic latitude of SSA $22\left(-44^{\circ}\right)$ compared with SSA $13\left(+74^{\circ}\right)$, which results in a larger fraction of stars.

Existing spectroscopy of a number of galaxies in these fields is summarized in Songaila et al. (1994). Most of the remaining objects without existing spectra were observed with the LRIS multi-object spectrograph (Oke et al. 1995) on the Keck $10 \mathrm{~m}$ telescope in a number of runs from 1994 August to 1995 September. Each mask on the spectrograph contained from 22 to 261 ." $4 \times 12^{\prime \prime}$ slits in the field area. The $300 \mathrm{l} / \mathrm{mm}$ grating used gives roughly $5000 \AA$ of wavelength coverage from $\sim 5000-10,000 \AA$ with a pixel size of 0.21 along the slit and $2.5 \AA$ in the wavelength direction. The blue wavelength cutoff for the multiple slits ranged in practice from $4300 \AA$ to $5700 \AA$, depending on the position of the slit on the mask. With the wide 1.4 slit, the resolution was $16 \AA$.

Because the CCD chip is extremely flat, we were able to adopt a relatively simple but robust observing procedure (a variant on self-flattening) which allowed us to avoid the extremely difficult and time-consuming problems of flatfielding, which is a considerable advantage because of internal flexure problems and the very high overheads associated with spectrograph setup, motions, and CCD readouts. Each mask was observed with objects positioned at the center of the slit and then at \pm 2.5 along the slit. Each such exposure was 20 minutes in duration giving a total integration on each mask of 1 hour. The minimum of the three frames at each pixel was used to perform a first-pass sky subtraction and then the frames were registered and median added to form the spectral image. A median $3 \times 3$ spatial filter was then used to identify and eliminate the very small number of cosmic rays present in the median summed frame. Next, the geometric distortion was removed from the spectral images using fits to the edge positions of the dispersed slits, as a function of wavelength. Third order polynomial fits to distortion-corrected observations of an Ar lamp taken at the beginning and end of the night were used to determine the wavelength scales, but, to allow for flexure, were offset to match the night-sky spectrum in each slit. A final linear sky subtraction in each slit completed the reduction. The night sky subtraction is generally excellent even at the redder wavelengths.

The final one-dimensional extraction was made using a summation weighted by the slit profile (optimal extraction) and was approximately flux calibrated to $f_{\nu}$ using observations of white dwarf standards taken in the same configuration. Redshifts were then measured for each of the objects by 
TABLE 1(a). Complete catalog SSA 13.

\begin{tabular}{|c|c|c|c|c|c|c|c|c|c|c|}
\hline \multirow{2}{*}{$\frac{\#}{0}$} & \multicolumn{3}{|c|}{ R.A. (1950) } & \multicolumn{3}{|c|}{ Dec. (1950) } & \multirow{2}{*}{$\frac{K}{18.4}$} & \multirow{2}{*}{$\frac{I}{20.9}$} & \multirow{2}{*}{$\frac{B}{22.9}$} & \multirow{2}{*}{$\frac{z}{0.839}$} \\
\hline & 13 & 10 & 17.04 & 42 & 59 & 39.9 & & & & \\
\hline 133 & 13 & 10 & 17.03 & 43 & 0 & 57.1 & 19.5 & 22.7 & 24.8 & 0.000 \\
\hline 2 & 13 & 10 & 16.19 & 42 & 59 & 26.0 & 17.2 & 20.4 & 23.8 & 0.378 \\
\hline 147 & 13 & 10 & 16.16 & 43 & 0 & 58.2 & 20.0 & 22.9 & 24.3 & 0.000 \\
\hline 3 & 13 & 10 & 15.79 & 42 & 59 & 34.0 & 19.8 & 21.8 & 23.6 & 0.799 \\
\hline 5 & 13 & 10 & 15.32 & 43 & 0 & 50.0 & 18.7 & 21.4 & 24.4 & 0.612 \\
\hline 134 & 13 & 10 & 15.25 & 43 & 0 & 13.9 & 19.7 & 22.9 & 25.2 & -1.000 \\
\hline 7 & 13 & 10 & 15.01 & 43 & 0 & 1.9 & 17.5 & 20.5 & 24.0 & 0.572 \\
\hline 8 & 13 & 10 & 14.77 & 42 & 59 & 52.1 & 19.4 & 21.4 & 23.4 & 0.572 \\
\hline 9 & 13 & 10 & 14.35 & 43 & 0 & 32.2 & 19.0 & 22.3 & 25.6 & 0.000 \\
\hline 12 & 13 & 10 & 13.73 & 42 & 59 & 52.2 & 16.1 & 19.0 & 22.6 & 0.489 \\
\hline 13 & 13 & 10 & 13.66 & 43 & 0 & 22.2 & 17.7 & 21.4 & 26.8 & -2.000 \\
\hline 14 & 13 & 10 & 13.58 & 43 & 0 & 4.2 & 19.8 & 22.3 & 24.5 & 0.667 \\
\hline 16 & 13 & 10 & 13.41 & 43 & 1 & 2.3 & 19.4 & 22.3 & 23.9 & 1.614 \\
\hline 17 & 13 & 10 & 13.32 & 42 & 59 & 42.8 & 18.4 & 20.8 & 24.7 & -2.000 \\
\hline 18 & 13 & 10 & 13.11 & 43 & 0 & 22.7 & 17.6 & 20.5 & 23.9 & 0.491 \\
\hline 19 & 13 & 10 & 13.08 & 43 & 0 & 45.0 & 18.9 & 20.8 & 22.8 & 0.393 \\
\hline 135 & 13 & 10 & 12.48 & 43 & 0 & 43.0 & 19.7 & 23.3 & 25.9 & -1.000 \\
\hline 21 & 13 & 10 & 11.94 & 42 & 59 & 59.1 & 19.2 & 21.1 & 22.9 & 0.443 \\
\hline 27 & 13 & 10 & 10.72 & 42 & 59 & 14.8 & 20.0 & 22.2 & 24.0 & 0.620 \\
\hline 136 & 13 & 10 & 10.61 & 42 & 59 & 39.0 & 19.6 & 23.7 & 25.3 & -1.000 \\
\hline 28 & 13 & 10 & 10.55 & 43 & 0 & 6.0 & 19.7 & 21.5 & 23.3 & 0.736 \\
\hline 29 & 13 & 10 & 10.30 & 42 & 59 & 38.8 & 18.5 & 21.7 & 24.2 & 1.038 \\
\hline 30 & 13 & 10 & 10.03 & 43 & 0 & 8.5 & 16.4 & 19.0 & 22.7 & -2.000 \\
\hline 137 & 13 & 10 & 9.78 & 43 & 1 & 4.7 & 19.6 & 23.0 & 25.6 & -1.000 \\
\hline 31 & 13 & 10 & 9.52 & 43 & 0 & 9.8 & 18.7 & 21.6 & 23.8 & 1.090 \\
\hline 138 & 13 & 10 & 9.30 & 42 & 59 & 50.8 & 19.4 & 24.2 & 26.1 & -1.000 \\
\hline 32 & 13 & 10 & 9.30 & 42 & 59 & 37.5 & 19.2 & 21.1 & 22.8 & 0.278 \\
\hline 155 & 13 & 10 & 9.25 & 42 & 59 & 24.1 & 20.0 & 22.6 & 24.2 & 0.730 \\
\hline 33 & 13 & 10 & 8.82 & 43 & 1 & 10.0 & 19.1 & 23.1 & -27.9 & 0.000 \\
\hline 34 & 13 & 10 & 8.64 & 42 & 59 & 13.7 & 18.8 & 21.5 & 23.9 & 0.417 \\
\hline 35 & 13 & 10 & 7.97 & 43 & 0 & 41.4 & 18.9 & 21.3 & 25.1 & -2.000 \\
\hline 36 & 13 & 10 & 7.86 & 43 & 0 & 53.5 & 18.8 & 20.8 & 22.7 & 0.338 \\
\hline 38 & 13 & 10 & 7.32 & 43 & 0 & 15.9 & 19.6 & 21.1 & 23.2 & 0.393 \\
\hline 39 & 13 & 10 & 7.28 & 43 & 0 & 6.7 & 18.2 & 20.3 & 22.3 & 0.449 \\
\hline 40 & 13 & 10 & 7.03 & 42 & 59 & 39.1 & 19.8 & 22.5 & 23.9 & 1.168 \\
\hline 41 & 13 & 10 & 6.77 & 43 & 0 & 32.2 & 19.1 & 21.6 & 24.7 & 0.480 \\
\hline 42 & 13 & 10 & 6.75 & 43 & 1 & 12.2 & 17.4 & 20.2 & 22.3 & 0.279 \\
\hline 43 & 13 & 10 & 6.68 & 42 & 59 & 59.5 & 19.1 & 22.1 & 23.6 & 1.305 \\
\hline 44 & 13 & 10 & 6.53 & 43 & 0 & 16.8 & 17.3 & 20.6 & 24.3 & 0.479 \\
\hline 45 & 13 & 10 & 6.48 & 43 & 0 & 3.9 & 19.7 & 21.5 & 25.2 & -1.000 \\
\hline 46 & 13 & 10 & 6.44 & 43 & 1 & 23.1 & 19.4 & 22.1 & 24.7 & 0.820 \\
\hline 47 & 13 & 10 & 6.17 & 43 & 0 & 27.3 & 17.4 & 20.3 & 24.5 & 0.732 \\
\hline 49 & 13 & 10 & 5.91 & 43 & 0 & 58.5 & 18.9 & 21.6 & 24.3 & 0.443 \\
\hline 50 & 13 & 10 & 5.83 & 42 & 59 & 29.9 & 19.6 & 22.1 & 24.9 & -1.000 \\
\hline 52 & 13 & 10 & 5.14 & 43 & 0 & 31.8 & 19.1 & 23.0 & 26.0 & 0.914 \\
\hline 54 & 13 & 10 & 4.72 & 43 & 1 & 5.4 & 19.5 & 22.4 & 25.7 & 0.000 \\
\hline 53 & 13 & 10 & 4.71 & 42 & 59 & 31.6 & 19.9 & 21.9 & 24.5 & 0.000 \\
\hline 56 & 13 & 10 & 4.48 & 43 & 1 & 23.8 & 19.2 & 22.0 & 24.3 & 0.394 \\
\hline 55 & 13 & 10 & 4.47 & 43 & 0 & 54.7 & 18.0 & 21.8 & 26.6 & 1.028 \\
\hline 57 & 13 & 10 & 4.19 & 42 & 59 & 34.3 & 18.5 & 21.1 & 24.1 & 0.318 \\
\hline 58 & 13 & 10 & 4.06 & 42 & 59 & 16.5 & 17.6 & 20.3 & 23.2 & 0.314 \\
\hline 59 & 13 & 10 & 3.95 & 43 & 0 & 49.6 & 19.4 & 22.9 & 24.4 & 1.483 \\
\hline 60 & 13 & 10 & 3.71 & 42 & 59 & 28.8 & 19.3 & 21.6 & 24.8 & 0.314 \\
\hline 62 & 13 & 10 & 3.20 & 42 & 59 & 38.5 & 15.8 & 18.7 & 22.1 & 0.314 \\
\hline 63 & 13 & 10 & 2.72 & 42 & 59 & 39.8 & 16.4 & 19.2 & 22.4 & 0.313 \\
\hline 64 & 13 & 10 & 2.53 & 43 & 0 & 6.0 & 18.8 & 21.5 & 23.9 & 0.681 \\
\hline 66 & 13 & 10 & 1.85 & 42 & 59 & 55.4 & 19.7 & 23.2 & 24.7 & -1.000 \\
\hline 69 & 13 & 10 & 0.91 & 42 & 59 & 16.2 & 19.1 & 21.4 & 23.3 & 0.317 \\
\hline 70 & 13 & 10 & 0.89 & 42 & 59 & 54.0 & 16.6 & 19.5 & 22.7 & 0.314 \\
\hline 71 & 13 & 10 & 0.79 & 43 & 0 & 18.8 & 19.3 & 21.9 & 24.5 & 0.210 \\
\hline 72 & 13 & 10 & 0.72 & 42 & 59 & 30.1 & 20.0 & 22.1 & 23.7 & 0.876 \\
\hline 73 & 13 & 9 & 59.98 & 43 & 1 & 24.4 & 19.7 & 22.2 & 25.8 & -2.000 \\
\hline 74 & 13 & 9 & 59.61 & 43 & 1 & 8.8 & 19.9 & 23.8 & 26.3 & 0.000 \\
\hline 75 & 13 & 9 & 59.61 & 43 & 1 & 1.7 & 19.9 & 22.4 & 24.5 & 0.818 \\
\hline 76 & 13 & 9 & 59.56 & 43 & 0 & 36.6 & 19.8 & 22.9 & 24.9 & 0.000 \\
\hline 77 & 13 & 9 & 59.25 & 42 & 59 & 50.0 & 19.3 & 25.5 & 27.1 & 0.000 \\
\hline 78 & 13 & 9 & 59.00 & 43 & 0 & 31.9 & 19.9 & 22.3 & 24.4 & 0.490 \\
\hline
\end{tabular}


TABLE 1(a). (continued)

\begin{tabular}{|c|c|c|c|c|c|c|c|c|c|c|}
\hline \multirow{2}{*}{$\frac{\#}{79}$} & \multicolumn{3}{|c|}{ R.A. (1950) } & \multicolumn{3}{|c|}{ Dec. (1950) } & \multirow{2}{*}{$\frac{K}{20.0}$} & \multirow{2}{*}{$\frac{I}{24.7}$} & \multirow{2}{*}{$\frac{B}{27.6}$} & \multirow{2}{*}{$\frac{z}{-1.000}$} \\
\hline & 13 & 9 & 58.87 & 43 & 0 & 10.9 & & & & \\
\hline 80 & 13 & 9 & 58.50 & 42 & 59 & 50.5 & 19.6 & 23.8 & 26.9 & 1.097 \\
\hline 81 & 13 & 9 & 58.00 & 42 & 59 & 17.2 & 19.7 & 21.4 & 22.9 & 0.082 \\
\hline 82 & 13 & 9 & 57.92 & 43 & 0 & 38.8 & 18.0 & 21.0 & 25.8 & -2.000 \\
\hline 83 & 13 & 9 & 56.89 & 42 & 59 & 14.2 & 19.0 & 21.6 & 23.7 & 0.669 \\
\hline 84 & 13 & 9 & 56.82 & 42 & 59 & 33.0 & 17.8 & 20.4 & 25.0 & -2.000 \\
\hline 85 & 13 & 9 & 56.79 & 43 & 0 & 7.2 & 18.4 & 20.9 & 21.9 & 0.950 \\
\hline 139 & 13 & 9 & 56.47 & 43 & 0 & 3.6 & 20.0 & 22.5 & 24.5 & 0.391 \\
\hline 91 & 13 & 9 & 55.56 & 43 & 0 & 19.1 & 16.5 & 19.0 & 22.4 & -2.000 \\
\hline 92 & 13 & 9 & 55.56 & 43 & 0 & 15.3 & 19.6 & 22.5 & 24.1 & 0.619 \\
\hline 140 & 13 & 9 & 53.38 & 43 & 0 & 47.7 & 19.6 & 23.2 & 24.9 & -1.000 \\
\hline 93 & 13 & 9 & 55.28 & 43 & 0 & 8.1 & 19.6 & 21.8 & 23.3 & 0.873 \\
\hline 94 & 13 & 9 & 54.73 & 43 & 0 & 31.1 & 19.1 & 20.5 & 21.6 & -2.000 \\
\hline 95 & 13 & 9 & 54.42 & 42 & 59 & 28.3 & 19.3 & 22.4 & 25.3 & 0.000 \\
\hline 96 & 13 & 9 & 54.23 & 42 & 59 & 53.8 & 19.6 & 21.2 & 22.9 & -2.000 \\
\hline 97 & 13 & 9 & 53.72 & 43 & 0 & 0.4 & 18.7 & 20.9 & 24.0 & -2.000 \\
\hline 98 & 13 & 9 & 53.66 & 42 & 59 & 24.7 & 19.3 & 21.6 & 23.7 & 0.923 \\
\hline 100 & 13 & 9 & 53.25 & 43 & 0 & 11.7 & 18.3 & 20.6 & 22.5 & 0.377 \\
\hline 141 & 13 & 9 & 52.15 & 43 & 0 & 53.1 & 18.9 & 22.9 & 25.4 & 0.619 \\
\hline 103 & 13 & 9 & 51.47 & 43 & 0 & 53.6 & 17.6 & 20.6 & 24.3 & 0.629 \\
\hline 105 & 13 & 9 & 50.55 & 42 & 59 & 30.1 & 18.8 & 21.3 & 24.2 & 0.395 \\
\hline 107 & 13 & 9 & 50.40 & 43 & 0 & 58.7 & 19.6 & 21.4 & 23.4 & 0.314 \\
\hline 108 & 13 & 9 & 50.19 & 42 & 59 & 14.7 & 18.6 & 21.1 & 23.5 & 0.680 \\
\hline 142 & 13 & 9 & 50.09 & 43 & 1 & 9.7 & 19.1 & 23.2 & 24.9 & 0.000 \\
\hline 109 & 13 & 9 & 50.08 & 42 & 59 & 42.4 & 18.5 & 20.9 & 23.7 & 0.393 \\
\hline 110 & 13 & 9 & 49.84 & 42 & 59 & 25.3 & 19.5 & 21.4 & 23.3 & 0.660 \\
\hline 111 & 13 & 9 & 49.75 & 43 & 0 & 27.7 & 18.0 & 21.2 & 25.8 & 0.729 \\
\hline 113 & 13 & 9 & 49.16 & 43 & 0 & 54.1 & 19.7 & 22.1 & 24.0 & 0.629 \\
\hline 112 & 13 & 9 & 49.15 & 42 & 59 & 17.9 & 20.0 & 21.6 & 23.4 & 0.764 \\
\hline 143 & 13 & 9 & 49.07 & 43 & 0 & 39.7 & 19.3 & 24.0 & 25.4 & -1.000 \\
\hline 144 & 13 & 9 & 49.03 & 43 & 0 & 34.5 & 19.5 & 24.2 & 27.3 & -1.000 \\
\hline 114 & 13 & 9 & 49.00 & 42 & 59 & 32.4 & 18.4 & 21.2 & 23.8 & 0.660 \\
\hline 115 & 13 & 9 & 48.69 & 43 & 1 & 3.8 & 19.4 & 22.3 & 25.0 & 0.389 \\
\hline 116 & 13 & 9 & 48.67 & 43 & 0 & 39.1 & 18.0 & 20.8 & 23.4 & 0.630 \\
\hline 145 & 13 & 9 & 48.43 & 43 & 0 & 25.8 & 19.1 & 23.2 & 24.9 & 0.840 \\
\hline 117 & 13 & 9 & 48.41 & 43 & 1 & 19.2 & 15.6 & 18.6 & 22.0 & 0.392 \\
\hline 118 & 13 & 9 & 47.79 & 42 & 59 & 13.6 & 18.8 & 21.5 & 23.3 & 0.761 \\
\hline 120 & 13 & 9 & 47.64 & 43 & 0 & 32.6 & 19.0 & 21.4 & 23.6 & 0.841 \\
\hline 121 & 13 & 9 & 47.29 & 42 & 59 & 40.6 & 17.2 & 20.0 & 23.1 & 0.392 \\
\hline 122 & 13 & 9 & 47.11 & 43 & 0 & 14.7 & 19.5 & 21.4 & 23.0 & 0.503 \\
\hline 146 & 13 & 9 & 46.96 & 42 & 59 & 24.8 & 18.8 & 23.7 & 24.7 & 0.781 \\
\hline 123 & 13 & 9 & 46.76 & 42 & 59 & 14.9 & 19.3 & 21.6 & 25.1 & 0.393 \\
\hline 124 & 13 & 9 & 46.73 & 43 & 0 & 56.4 & 15.7 & 18.7 & 22.4 & 0.393 \\
\hline 125 & 13 & 9 & 46.61 & 43 & 1 & 2.1 & 18.9 & 22.0 & 25.4 & 0.000 \\
\hline 126 & 13 & 9 & 46.21 & 42 & 59 & 44.9 & 16.5 & 19.0 & 21.2 & 0.177 \\
\hline 127 & 13 & 9 & 46.21 & 43 & 1 & 0.2 & 19.5 & 22.5 & 25.0 & 0.393 \\
\hline 129 & 13 & 9 & 44.96 & 43 & 0 & 29.8 & 19.8 & 22.5 & 23.9 & 0.000 \\
\hline 130 & 13 & 9 & 44.86 & 42 & 59 & 31.9 & 19.6 & 21.5 & 22.4 & 0.315 \\
\hline 132 & 13 & 9 & 43.95 & 43 & 1 & 17.1 & 19.2 & 20.9 & 22.5 & 0.275 \\
\hline 1 & 13 & 10 & 16.93 & 43 & 0 & 3.4 & 20.0 & 22.0 & 23.2 & 1.041 \\
\hline 4 & 13 & 10 & 15.55 & 42 & 59 & 31.7 & 20.4 & 22.4 & 24.4 & 0.909 \\
\hline 6 & 13 & 10 & 15.08 & 42 & 59 & 38.6 & 20.7 & 22.4 & 24.2 & 0.799 \\
\hline 10 & 13 & 10 & 14.35 & 43 & 0 & 35.5 & 20.4 & 21.7 & 23.3 & 0.554 \\
\hline 11 & 13 & 10 & 13.87 & 43 & 0 & 39.7 & 20.3 & 22.5 & 24.1 & 1.225 \\
\hline 15 & 13 & 10 & 13.52 & 42 & 59 & 25.5 & 20.6 & 22.5 & 24.5 & 0.443 \\
\hline 20 & 13 & 10 & 12.58 & 43 & 0 & 10.0 & 20.3 & 22.3 & 23.7 & 1.028 \\
\hline 22 & 13 & 10 & 11.88 & 42 & 59 & 18.0 & 20.5 & 22.2 & 24.7 & -2.000 \\
\hline 23 & 13 & 10 & 11.56 & 42 & 59 & 26.7 & 20.3 & 22.1 & 24.3 & 0.680 \\
\hline 24 & 13 & 10 & 11.46 & 42 & 59 & 56.3 & 20.1 & 22.1 & 23.3 & 1.141 \\
\hline 25 & 13 & 10 & 11.42 & 43 & 0 & 7.7 & 20.4 & 22.2 & 24.4 & 0.733 \\
\hline 37 & 13 & 10 & 7.59 & 43 & 1 & 4.0 & 20.1 & 22.5 & 24.5 & 1.028 \\
\hline 48 & 13 & 10 & 5.91 & 43 & 1 & 5.4 & 20.2 & 22.1 & 24.3 & 0.489 \\
\hline 51 & 13 & 10 & 5.84 & 42 & 59 & 46.8 & 20.2 & 22.2 & 23.5 & 1.156 \\
\hline 61 & 13 & 10 & 3.72 & 42 & 59 & 49.5 & 21.0 & 22.2 & 23.9 & 0.315 \\
\hline 67 & 13 & 10 & 1.40 & 43 & 0 & 53.6 & 20.1 & 22.0 & 23.9 & 0.274 \\
\hline 68 & 13 & 10 & 1.07 & 43 & 0 & 32.9 & 20.4 & 22.4 & 24.2 & -1.000 \\
\hline 86 & 13 & 9 & 56.75 & 43 & 1 & 17.5 & 20.2 & 21.9 & 23.6 & 0.000 \\
\hline
\end{tabular}


TABLE 1(a). (continued)

\begin{tabular}{|c|c|c|c|c|c|c|c|c|c|c|}
\hline \multirow{2}{*}{$\frac{\#}{88}$} & \multicolumn{3}{|c|}{ R.A. (1950) } & \multicolumn{3}{|c|}{ Dec. (1950) } & \multirow{2}{*}{$\frac{K}{20.2}$} & \multirow{2}{*}{$\frac{I}{21.7}$} & \multirow{2}{*}{$\frac{B}{23.3}$} & \multirow{2}{*}{$\frac{z}{0.217}$} \\
\hline & 13 & 9 & 56.18 & 43 & 0 & 57.1 & & & & \\
\hline 99 & 13 & 9 & 53.66 & 43 & 0 & 36.5 & 20.4 & 22.3 & 23.7 & 1.368 \\
\hline 106 & 13 & 9 & 50.55 & 43 & 0 & 42.1 & 20.5 & 22.5 & 24.4 & 0.000 \\
\hline 119 & 13 & 9 & 47.67 & 43 & 0 & 24.1 & 20.7 & 21.8 & 23.3 & -2.000 \\
\hline 128 & 13 & 9 & 45.66 & 43 & 1 & 4.7 & 20.1 & 22.1 & 23.4 & 0.180 \\
\hline 148 & 13 & 10 & 15.83 & 43 & 0 & 40.6 & 21.0 & 23.1 & 24.4 & -1.000 \\
\hline 149 & 13 & 10 & 15.12 & 43 & 0 & 59.5 & 22.4 & 24.3 & 24.3 & -1.000 \\
\hline 150 & 13 & 10 & 12.54 & 42 & 59 & 56.5 & 20.7 & 22.9 & 24.5 & -1.000 \\
\hline 151 & 13 & 10 & 12.29 & 43 & 0 & 49.8 & 21.4 & 23.2 & 24.0 & 0.000 \\
\hline 152 & 13 & 10 & 11.53 & 42 & 59 & 31.2 & 22.3 & 24.0 & 24.4 & -1.000 \\
\hline 26 & 13 & 10 & 11.29 & 42 & 59 & 29.1 & 21.0 & 22.9 & 24.4 & 1.503 \\
\hline 153 & 13 & 10 & 10.48 & 43 & 0 & 30.0 & 21.4 & 23.1 & 24.3 & 0.087 \\
\hline 154 & 13 & 10 & 9.25 & 42 & 59 & 28.2 & 23.0 & 23.3 & 24.1 & 0.000 \\
\hline 156 & 13 & 10 & 5.47 & 42 & 59 & 38.9 & 20.5 & 24.1 & 24.5 & -1.000 \\
\hline 175 & 13 & 10 & 4.74 & 43 & 0 & 37.8 & 20.5 & 23.7 & 24.5 & -1.000 \\
\hline 157 & 13 & 10 & 4.35 & 42 & 59 & 25.6 & -24.1 & 24.2 & 24.4 & -1.000 \\
\hline 158 & 13 & 10 & 2.85 & 43 & 0 & 1.3 & 21.4 & 22.7 & 24.3 & 0.505 \\
\hline 159 & 13 & 10 & 2.01 & 42 & 59 & 50.5 & 21.2 & 23.8 & 24.4 & -1.000 \\
\hline 160 & 13 & 10 & 1.66 & 43 & 0 & 20.5 & 22.1 & 23.2 & 24.5 & 0.402 \\
\hline 161 & 13 & 10 & 1.64 & 43 & 0 & 45.9 & 20.1 & 23.2 & 24.3 & 0.000 \\
\hline 162 & 13 & 10 & 0.66 & 43 & 0 & 5.5 & 21.7 & 23.0 & 24.3 & 0.873 \\
\hline 163 & 13 & 9 & 57.57 & 43 & 0 & 47.9 & 20.8 & 23.3 & 24.5 & 0.000 \\
\hline 164 & 13 & 9 & 56.97 & 42 & 59 & 21.8 & 20.9 & 23.6 & 24.1 & 0.000 \\
\hline 87 & 13 & 9 & 56.50 & 42 & 59 & 36.4 & 20.2 & 22.5 & 23.6 & 1.427 \\
\hline 89 & 13 & 9 & 55.96 & 43 & 0 & 10.2 & 22.0 & 22.5 & 23.8 & 0.951 \\
\hline 90 & 13 & 9 & 55.88 & 43 & 0 & 3.2 & 22.7 & 23.1 & 23.6 & 1.247 \\
\hline 165 & 13 & 9 & 55.42 & 42 & 59 & 32.6 & 24.3 & 23.8 & 24.2 & -1.000 \\
\hline 174 & 13 & 9 & 54.62 & 42 & 59 & 27.0 & 20.6 & 22.7 & 24.5 & 0.479 \\
\hline 166 & 13 & 9 & 54.02 & 43 & 0 & 42.7 & 21.1 & 24.1 & 24.4 & -1.000 \\
\hline 167 & 13 & 9 & 52.58 & 43 & 0 & 30.5 & 20.0 & 23.5 & 24.2 & -1.000 \\
\hline 101 & 13 & 9 & 52.05 & 43 & 0 & 12.8 & 20.3 & 22.9 & 24.0 & 1.256 \\
\hline 102 & 13 & 9 & 51.58 & 43 & 0 & 29.7 & 22.0 & 23.3 & 23.9 & 1.496 \\
\hline 168 & 13 & 9 & 51.34 & 43 & 0 & 22.7 & 20.8 & 24.0 & 24.5 & 0.000 \\
\hline 104 & 13 & 9 & 51.14 & 43 & 0 & 28.3 & 20.8 & 23.6 & 24.0 & 0.000 \\
\hline 169 & 13 & 9 & 50.53 & 43 & 0 & 4.5 & 20.9 & 23.7 & 24.3 & -1.000 \\
\hline 170 & 13 & 9 & 49.80 & 43 & 0 & 56.7 & -22.1 & 23.7 & 24.2 & -1.000 \\
\hline 171 & 13 & 9 & 49.35 & 43 & 0 & 27.5 & 21.2 & 22.9 & 24.5 & 0.726 \\
\hline 172 & 13 & 9 & 47.61 & 43 & 1 & 5.0 & 20.4 & 23.4 & 24.5 & 0.000 \\
\hline 173 & 13 & 9 & 46.88 & 43 & 0 & 27.1 & 20.4 & 23.2 & 24.3 & -1.000 \\
\hline
\end{tabular}

Notes to TABLE 1a

The SSA13 sample selected to $K=20, I=23$, and $B=24.5$. Objects with redshifts designated -1.000 were not observed; those with redshifts designated -2.000 were identified as stars. 
TABle 1(b). Complete catalog SSA 22.

\begin{tabular}{|c|c|c|c|c|c|c|c|c|c|c|}
\hline \multirow{2}{*}{$\frac{\#}{0}$} & \multicolumn{3}{|c|}{ R.A. (1950) } & \multicolumn{3}{|c|}{ Dec. (1950) } & \multirow{2}{*}{$\frac{K}{18.2}$} & \multirow{2}{*}{$\frac{I}{20.0}$} & \multirow{2}{*}{$\frac{B}{21.7}$} & \multirow{2}{*}{$\frac{z}{0.229}$} \\
\hline & 22 & 15 & 10.56 & 0 & 0 & 3.3 & & & & \\
\hline 1 & 22 & 15 & 10.57 & 0 & 1 & 3.1 & 18.4 & 20.4 & 22.2 & 0.356 \\
\hline 2 & 22 & 15 & 10.43 & -0 & 0 & 51.8 & 17.9 & 19.1 & 21.1 & -2.000 \\
\hline 3 & 22 & 15 & 10.15 & -0 & 0 & 40.9 & 14.3 & 16.8 & 16.8 & -2.000 \\
\hline 4 & 22 & 15 & 10.13 & -0 & 1 & 8.8 & 19.8 & 23.5 & 24.9 & -1.000 \\
\hline 8 & 22 & 15 & 9.74 & 0 & 0 & 40.8 & 16.4 & 18.6 & 23.0 & -2.000 \\
\hline 10 & 22 & 15 & 9.52 & -0 & 1 & 13.5 & 17.7 & 20.3 & 23.9 & 0.514 \\
\hline 12 & 22 & 15 & 9.37 & 0 & 0 & 23.4 & 17.9 & 20.1 & 24.5 & -2.000 \\
\hline 14 & 22 & 15 & 9.34 & 0 & 0 & 6.1 & 19.1 & 21.4 & 23.8 & 0.538 \\
\hline 15 & 22 & 15 & 9.31 & -0 & 0 & 11.0 & 19.0 & 23.4 & 25.7 & 0.000 \\
\hline 16 & 22 & 15 & 9.27 & -0 & 0 & 49.3 & 17.1 & 18.3 & 19.6 & -2.000 \\
\hline 17 & 22 & 15 & 9.11 & 0 & 0 & 7.9 & 18.7 & 20.8 & 22.8 & 0.966 \\
\hline 18 & 22 & 15 & 8.83 & -0 & 1 & 13.6 & 18.2 & 20.9 & 24.4 & 0.514 \\
\hline 19 & 22 & 15 & 8.77 & -0 & 0 & 40.7 & 18.1 & 20.0 & 22.0 & 0.294 \\
\hline 20 & 22 & 15 & 8.77 & -0 & 0 & 16.2 & 19.6 & 23.0 & 26.0 & 0.663 \\
\hline 21 & 22 & 15 & 8.59 & 0 & 0 & 32.6 & 14.5 & 17.3 & 19.3 & -2.000 \\
\hline 22 & 22 & 15 & 8.51 & -0 & 0 & 36.9 & 19.6 & 23.7 & 32.0 & 0.000 \\
\hline 23 & 22 & 15 & 8.35 & -0 & 0 & 31.2 & 19.4 & 23.2 & 27.7 & 0.000 \\
\hline 24 & 22 & 15 & 8.32 & -0 & 1 & 2.9 & 19.1 & 21.7 & -26.6 & -2.000 \\
\hline 25 & 22 & 15 & 8.09 & 0 & 0 & 59.5 & 19.8 & 23.5 & 25.0 & 0.000 \\
\hline 26 & 22 & 15 & 7.83 & -0 & 1 & 8.0 & 18.4 & 20.8 & 23.1 & 0.521 \\
\hline 27 & 22 & 15 & 7.80 & -0 & 0 & 49.9 & 14.0 & 16.7 & 18.1 & -2.000 \\
\hline 28 & 22 & 15 & 7.65 & 0 & 0 & 21.2 & 16.3 & 18.7 & 21.2 & 0.247 \\
\hline 30 & 22 & 15 & 7.45 & -0 & 0 & 29.3 & 18.5 & 21.4 & 26.0 & 0.751 \\
\hline 32 & 22 & 15 & 7.36 & -0 & 0 & 50.1 & 18.4 & 21.4 & 24.3 & 1.024 \\
\hline 33 & 22 & 15 & 7.34 & -0 & 0 & 45.1 & 19.6 & 21.7 & 24.5 & 0.707 \\
\hline 34 & 22 & 15 & 7.33 & -0 & 0 & 0.7 & 19.6 & 24.2 & 27.1 & -1.000 \\
\hline 35 & 22 & 15 & 7.34 & 0 & 0 & 14.7 & 14.1 & 17.1 & 19.4 & -2.000 \\
\hline 37 & 22 & 15 & 6.95 & 0 & 0 & 46.2 & 19.4 & 21.4 & 25.7 & -2.000 \\
\hline 39 & 22 & 15 & 6.74 & 0 & 0 & 28.2 & 19.7 & 22.6 & 24.2 & 1.205 \\
\hline 40 & 22 & 15 & 6.65 & -0 & 0 & 58.7 & 18.2 & 21.2 & 25.3 & 0.707 \\
\hline 41 & 22 & 15 & 6.62 & -0 & 0 & 54.4 & 19.3 & 21.0 & 24.9 & -2.000 \\
\hline 44 & 22 & 15 & 6.37 & 0 & 0 & 7.4 & 18.4 & 21.1 & 25.0 & 0.672 \\
\hline 45 & 22 & 15 & 6.23 & -0 & 0 & 24.0 & 18.9 & 20.6 & 22.3 & 0.132 \\
\hline 46 & 22 & 15 & 6.25 & 0 & 0 & 10.0 & 18.6 & 21.5 & 24.3 & 0.912 \\
\hline 49 & 22 & 15 & 6.03 & -0 & 0 & 13.3 & 19.9 & 22.0 & 23.9 & 0.707 \\
\hline 50 & 22 & 15 & 5.94 & -0 & 0 & 17.8 & 18.8 & 20.8 & 22.9 & 0.538 \\
\hline 51 & 22 & 15 & 5.78 & 0 & 0 & 23.0 & 18.7 & 20.9 & 22.7 & 0.536 \\
\hline 53 & 22 & 15 & 5.61 & -0 & 1 & 8.0 & 18.9 & 20.3 & 23.1 & -2.000 \\
\hline 54 & 22 & 15 & 5.56 & 0 & 0 & 30.6 & 18.9 & 20.7 & 23.1 & 0.418 \\
\hline 55 & 22 & 15 & 5.43 & 0 & 0 & 33.9 & 18.8 & 21.6 & 24.0 & 0.815 \\
\hline 59 & 22 & 15 & 4.89 & 0 & 0 & 3.4 & 18.5 & 21.0 & 24.3 & 0.418 \\
\hline 60 & 22 & 15 & 4.89 & 0 & 0 & 38.7 & 19.2 & 23.0 & 25.1 & 1.392 \\
\hline 61 & 22 & 15 & 4.88 & 0 & 0 & 56.0 & 18.8 & 20.8 & 25.5 & -2.000 \\
\hline 62 & 22 & 15 & 4.76 & -0 & 0 & 17.4 & 15.7 & 18.1 & 21.4 & -2.000 \\
\hline 63 & 22 & 15 & 4.15 & 0 & 0 & 18.0 & 17.8 & 19.0 & 20.7 & -2.000 \\
\hline 64 & 22 & 15 & 3.95 & 0 & 0 & 56.6 & 17.6 & 21.3 & 25.7 & 0.653 \\
\hline 65 & 22 & 15 & 3.95 & 0 & 1 & 3.8 & 19.9 & 23.0 & 25.2 & 0.000 \\
\hline 66 & 22 & 15 & 3.71 & 0 & 1 & 0.2 & 19.3 & 22.5 & 24.2 & 0.875 \\
\hline 69 & 22 & 15 & 3.49 & -0 & 0 & 0.8 & 19.7 & 22.0 & 25.1 & 0.692 \\
\hline 70 & 22 & 15 & 3.24 & -0 & 0 & 55.0 & 18.0 & 20.4 & 23.5 & 0.348 \\
\hline 72 & 22 & 15 & 3.09 & 0 & 0 & 25.8 & 19.9 & 22.0 & 23.8 & 0.787 \\
\hline 73 & 22 & 15 & 2.96 & -0 & 0 & 36.2 & 18.3 & 21.4 & 25.5 & 0.822 \\
\hline 77 & 22 & 15 & 2.06 & -0 & 1 & 9.5 & 18.0 & 22.2 & 25.8 & 1.020 \\
\hline 78 & 22 & 15 & 2.07 & 0 & 0 & 23.9 & 19.0 & 22.2 & 26.1 & 0.823 \\
\hline 81 & 22 & 15 & 1.66 & -0 & 0 & 23.3 & 19.7 & 21.7 & 23.5 & 0.384 \\
\hline 82 & 22 & 15 & 1.63 & -0 & 0 & 27.8 & 19.2 & 21.0 & 22.7 & 0.384 \\
\hline 83 & 22 & 15 & 1.36 & -0 & 0 & 32.0 & 19.9 & 21.7 & 23.5 & 0.510 \\
\hline 84 & 22 & 15 & 1.20 & -0 & 0 & 1.1 & 16.5 & 19.1 & 22.3 & 0.301 \\
\hline 85 & 22 & 15 & 1.14 & -0 & 1 & 11.7 & 19.8 & 21.8 & 23.3 & 1.010 \\
\hline 86 & 22 & 15 & 1.14 & 0 & 0 & 15.4 & 19.0 & 20.7 & 23.9 & -2.000 \\
\hline 87 & 22 & 15 & 0.96 & -0 & 0 & 32.9 & 19.6 & 21.7 & 24.1 & 0.306 \\
\hline 88 & 22 & 15 & 0.90 & -0 & 0 & 30.1 & 19.1 & 21.1 & 25.1 & -2.000 \\
\hline 89 & 22 & 15 & 0.87 & -0 & 0 & 12.0 & 19.7 & 22.4 & 23.7 & 1.151 \\
\hline 90 & 22 & 15 & 0.82 & -0 & 0 & 24.8 & 19.9 & 22.3 & 25.3 & 0.419 \\
\hline 92 & 22 & 15 & 0.29 & 0 & 0 & 9.3 & 17.9 & 20.4 & 23.7 & 0.381 \\
\hline 93 & 22 & 15 & 0.26 & -0 & 1 & 10.1 & 19.3 & 21.6 & 24.1 & 0.377 \\
\hline 94 & 22 & 15 & 0.17 & -0 & 0 & 10.6 & 19.9 & 23.8 & 27.0 & 0.000 \\
\hline
\end{tabular}


TABLE 1(b). (continued)

\begin{tabular}{|c|c|c|c|c|c|c|c|c|c|c|}
\hline \multirow{2}{*}{$\frac{\#}{95}$} & \multicolumn{3}{|c|}{ R.A. (1950) } & \multicolumn{3}{|c|}{ Dec. (1950) } & \multirow{2}{*}{$\frac{K}{17.1}$} & \multirow{2}{*}{$\frac{I}{18.8}$} & \multirow{2}{*}{$\frac{B}{22.3}$} & \multirow{2}{*}{$\frac{z}{-2.000}$} \\
\hline & 22 & 14 & 59.96 & -0 & 0 & 53.1 & & & & \\
\hline 96 & 22 & 14 & 59.93 & -0 & 0 & 36.1 & 15.9 & 18.6 & 22.0 & 0.290 \\
\hline 98 & 22 & 14 & 59.80 & -0 & 0 & 43.3 & 19.0 & 21.9 & 23.7 & 1.010 \\
\hline 100 & 22 & 14 & 59.49 & -0 & 0 & 29.5 & 18.6 & 21.0 & 23.8 & 0.303 \\
\hline 101 & 22 & 14 & 59.49 & -0 & 0 & 33.8 & 20.0 & 24.0 & 25.3 & -1.000 \\
\hline 102 & 22 & 14 & 59.47 & 0 & 0 & 47.2 & 17.9 & 21.1 & 25.2 & 0.824 \\
\hline 104 & 22 & 14 & 59.25 & 0 & 0 & 21.3 & 19.4 & 22.5 & 26.7 & 0.000 \\
\hline 106 & 22 & 14 & 59.16 & -0 & 1 & 2.3 & 19.2 & 21.9 & 24.2 & 0.616 \\
\hline 107 & 22 & 14 & 59.14 & -0 & 0 & 13.9 & 18.8 & 23.3 & 25.7 & 0.000 \\
\hline 108 & 22 & 14 & 59.13 & 0 & 0 & 42.1 & 18.8 & 21.0 & 23.0 & 0.588 \\
\hline 109 & 22 & 14 & 59.08 & -0 & 0 & 35.6 & 16.4 & 18.6 & 22.9 & -2.000 \\
\hline 110 & 22 & 14 & 58.88 & 0 & 0 & 38.5 & 18.4 & 20.1 & 23.5 & -2.000 \\
\hline 111 & 22 & 14 & 58.78 & -0 & 0 & 42.2 & 19.9 & 22.2 & 25.1 & 0.302 \\
\hline 112 & 22 & 14 & 58.52 & -0 & 0 & 14.0 & 18.8 & 21.4 & 26.6 & -2.000 \\
\hline 114 & 22 & 14 & 58.38 & 0 & 0 & 36.5 & 17.2 & 18.4 & 19.9 & -2.000 \\
\hline 116 & 22 & 14 & 58.21 & -0 & 0 & 26.0 & 18.0 & 20.4 & 24.6 & -2.000 \\
\hline 117 & 22 & 14 & 58.13 & -0 & 0 & 54.0 & 14.9 & 17.9 & 22.2 & -2.000 \\
\hline 118 & 22 & 14 & 58.01 & 0 & 0 & 9.7 & 19.1 & 22.1 & 25.8 & 0.816 \\
\hline 120 & 22 & 14 & 57.58 & -0 & 0 & 42.5 & 18.3 & 20.4 & 24.2 & -2.000 \\
\hline 122 & 22 & 14 & 57.35 & 0 & 0 & 54.8 & 19.8 & 22.4 & 24.6 & 1.625 \\
\hline 123 & 22 & 14 & 56.93 & 0 & 1 & 1.6 & 17.1 & 18.9 & 20.5 & 0.095 \\
\hline 124 & 22 & 14 & 56.81 & 0 & 0 & 26.6 & 19.8 & 21.8 & 23.5 & 0.671 \\
\hline 125 & 22 & 14 & 56.76 & -0 & 0 & 14.9 & 18.6 & 21.7 & 24.7 & 0.873 \\
\hline 129 & 22 & 14 & 55.91 & -0 & 0 & 59.0 & 16.3 & 18.0 & 18.8 & -2.000 \\
\hline 130 & 22 & 14 & 55.61 & 0 & 0 & 26.9 & 18.9 & 20.6 & 24.1 & -2.000 \\
\hline 131 & 22 & 14 & 55.51 & -0 & 0 & 39.1 & 16.0 & 18.4 & 22.7 & -2.000 \\
\hline 134 & 22 & 14 & 55.06 & 0 & 0 & 28.2 & 15.2 & 17.2 & 17.4 & -2.000 \\
\hline 135 & 22 & 14 & 55.03 & 0 & 0 & 47.9 & 20.0 & 24.2 & 26.7 & -1.000 \\
\hline 136 & 22 & 14 & 55.01 & -0 & 0 & 25.8 & 19.9 & 23.0 & 25.3 & 0.000 \\
\hline 137 & 22 & 14 & 55.07 & 0 & 1 & 2.7 & 19.6 & 23.1 & 23.8 & 0.000 \\
\hline 138 & 22 & 14 & 54.97 & 0 & 1 & 6.5 & 18.1 & 19.8 & 22.5 & -2.000 \\
\hline 140 & 22 & 14 & 54.58 & -0 & 0 & 34.1 & 19.3 & 23.1 & 26.5 & 0.000 \\
\hline 143 & 22 & 14 & 54.45 & 0 & 0 & 0.6 & 18.1 & 21.9 & 24.6 & 1.102 \\
\hline 144 & 22 & 14 & 54.21 & -0 & 0 & 28.7 & 18.4 & 22.3 & 26.4 & 0.000 \\
\hline 145 & 22 & 14 & 54.16 & -0 & 0 & 4.0 & 19.5 & 23.0 & 27.0 & 0.000 \\
\hline 146 & 22 & 14 & 53.75 & -0 & 0 & 31.1 & 18.7 & 22.2 & 24.8 & 0.000 \\
\hline 147 & 22 & 14 & 53.59 & -0 & 0 & 51.4 & 18.7 & 21.3 & 23.8 & 0.514 \\
\hline 148 & 22 & 14 & 53.57 & -0 & 0 & 24.5 & 17.7 & 21.0 & 25.6 & 0.876 \\
\hline 149 & 22 & 14 & 53.56 & 0 & 1 & 2.1 & 19.3 & 20.8 & 22.9 & -2.000 \\
\hline 150 & 22 & 14 & 53.17 & -0 & 0 & 37.4 & 19.0 & 22.2 & 25.6 & 0.795 \\
\hline 151 & 22 & 14 & 52.99 & -0 & 0 & 55.4 & 16.6 & 18.6 & 22.6 & -2.000 \\
\hline 152 & 22 & 14 & 52.83 & 0 & 0 & 9.2 & 17.3 & 20.3 & 23.6 & 0.617 \\
\hline 153 & 22 & 14 & 52.80 & -0 & 0 & 6.4 & 17.7 & 20.0 & 24.6 & -2.000 \\
\hline 154 & 22 & 14 & 52.76 & 0 & 0 & 1.7 & 18.4 & 21.1 & 23.8 & 0.614 \\
\hline 157 & 22 & 14 & 52.42 & -0 & 0 & 29.2 & 19.7 & 21.7 & 25.1 & -2.000 \\
\hline 158 & 22 & 14 & 52.23 & 0 & 1 & 2.2 & 17.7 & 19.4 & 22.2 & -2.000 \\
\hline 161 & 22 & 14 & 52.07 & 0 & 0 & 5.2 & 19.5 & 22.7 & 27.2 & 0.960 \\
\hline 162 & 22 & 14 & 51.98 & 0 & 0 & 55.8 & 16.8 & 18.4 & 19.9 & -2.000 \\
\hline 165 & 22 & 14 & 51.42 & 0 & 0 & 52.6 & 19.5 & 22.8 & 28.2 & 0.795 \\
\hline 166 & 22 & 14 & 51.40 & 0 & 0 & 7.3 & 19.5 & 22.8 & 26.9 & 0.378 \\
\hline 168 & 22 & 14 & 51.24 & -0 & 0 & 11.2 & 19.2 & 21.8 & 24.4 & 0.408 \\
\hline 169 & 22 & 14 & 51.24 & 0 & 0 & 38.1 & 18.9 & 20.7 & 24.3 & -2.000 \\
\hline 170 & 22 & 14 & 51.17 & 0 & 0 & 57.9 & 18.3 & 21.8 & 24.5 & 0.960 \\
\hline 171 & 22 & 14 & 51.08 & -0 & 1 & 2.9 & 17.6 & 19.5 & 23.8 & -2.000 \\
\hline 172 & 22 & 14 & 51.03 & 0 & 0 & 7.0 & 18.5 & 21.2 & 23.1 & 0.378 \\
\hline 173 & 22 & 14 & 50.92 & -0 & 0 & 53.9 & 19.4 & 23.3 & 27.5 & -1.000 \\
\hline 174 & 22 & 14 & 50.55 & -0 & 1 & 6.4 & 19.8 & 23.6 & 26.8 & 0.000 \\
\hline 179 & 22 & 14 & 49.93 & 0 & 0 & 33.7 & 18.9 & 21.6 & 24.8 & 0.670 \\
\hline 180 & 22 & 14 & 49.80 & -0 & 0 & 17.1 & 17.1 & 18.8 & 21.6 & -2.000 \\
\hline 183 & 22 & 14 & 49.53 & -0 & 0 & 4.5 & 18.0 & 21.0 & 24.3 & 0.476 \\
\hline 184 & 22 & 14 & 49.38 & -0 & 1 & 9.1 & 19.4 & 20.9 & 22.3 & 0.214 \\
\hline 185 & 22 & 14 & 49.39 & -0 & 0 & 27.7 & 17.9 & 20.3 & 22.8 & 0.317 \\
\hline 186 & 22 & 14 & 49.32 & 0 & 0 & 59.5 & 19.3 & 23.2 & -26.3 & 0.000 \\
\hline 187 & 22 & 14 & 48.36 & -0 & 0 & 19.7 & 19.6 & 24.1 & 24.3 & 0.000 \\
\hline 188 & 22 & 14 & 48.37 & 0 & 1 & 2.5 & 19.6 & 31.1 & 26.2 & 0.000 \\
\hline 190 & 22 & 14 & 47.85 & -0 & 0 & 10.0 & 18.7 & 22.4 & 25.1 & 0.000 \\
\hline 191 & 22 & 14 & 47.83 & 0 & 0 & 10.0 & 17.7 & 20.2 & 22.6 & 0.279 \\
\hline 194 & 22 & 14 & 47.38 & 0 & 0 & 21.7 & 18.1 & 20.6 & 23.8 & 0.471 \\
\hline
\end{tabular}


TABLE 1(b). (continued)

\begin{tabular}{|c|c|c|c|c|c|c|c|c|c|c|}
\hline \multirow{2}{*}{$\frac{\#}{197}$} & \multicolumn{3}{|c|}{ R.A. (1950) } & \multicolumn{3}{|c|}{ Dec. $(1950)$} & \multirow{2}{*}{$\frac{K}{19.4}$} & \multirow{2}{*}{$\frac{I}{21.8}$} & \multirow{2}{*}{$\frac{B}{-28.3}$} & \multirow{2}{*}{$\frac{z}{-2.000}$} \\
\hline & 22 & 15 & 7.83 & -0 & 1 & 4.6 & & & & \\
\hline 5 & 22 & 15 & 9.96 & 0 & 0 & 54.4 & 20.1 & 22.5 & 24.4 & 0.426 \\
\hline 6 & 22 & 15 & 9.87 & -0 & 0 & 51.9 & -23.5 & 22.9 & 24.6 & 0.188 \\
\hline 7 & 22 & 15 & 9.83 & 0 & 1 & 4.0 & 20.1 & 22.5 & 24.2 & 0.768 \\
\hline 9 & 22 & 15 & 9.60 & -0 & 0 & 21.7 & 20.0 & 22.2 & 23.7 & 1.093 \\
\hline 11 & 22 & 15 & 9.54 & -0 & 0 & 1.6 & 20.2 & 22.2 & 24.0 & 0.626 \\
\hline 13 & 22 & 15 & 9.38 & -0 & 0 & 2.4 & 21.3 & 22.4 & 24.6 & 0.653 \\
\hline 36 & 22 & 15 & 7.23 & 0 & 0 & 41.1 & 20.7 & 23.0 & 24.2 & 0.000 \\
\hline 42 & 22 & 15 & 6.44 & -0 & 0 & 22.1 & 21.3 & 22.3 & 23.9 & -2.000 \\
\hline 43 & 22 & 15 & 6.36 & -0 & 1 & 6.6 & 20.1 & 21.4 & 22.9 & 0.198 \\
\hline 47 & 22 & 15 & 6.11 & 0 & 0 & 1.0 & 21.2 & 22.1 & 23.4 & 0.173 \\
\hline 48 & 22 & 15 & 6.11 & 0 & 0 & 24.9 & 20.7 & 22.9 & 24.1 & 0.000 \\
\hline 52 & 22 & 15 & 5.75 & -0 & 1 & 10.9 & 21.2 & 22.7 & 24.4 & 0.794 \\
\hline 67 & 22 & 15 & 3.67 & 0 & 0 & 42.4 & 20.9 & 22.8 & 24.5 & 0.588 \\
\hline 68 & 22 & 15 & 3.59 & 0 & 0 & 51.6 & 20.7 & 22.9 & 23.9 & 1.560 \\
\hline 71 & 22 & 15 & 3.17 & -0 & 0 & 20.4 & 21.2 & 22.1 & 23.1 & 0.132 \\
\hline 74 & 22 & 15 & 2.87 & -0 & 0 & 20.7 & 20.4 & 22.8 & 23.3 & 1.360 \\
\hline 75 & 22 & 15 & 2.35 & 0 & 0 & 14.7 & 20.2 & 22.7 & 24.2 & 0.724 \\
\hline 80 & 22 & 15 & 1.70 & 0 & 0 & 29.5 & 21.8 & 22.2 & 23.3 & 1.669 \\
\hline 91 & 22 & 15 & 0.48 & 0 & 0 & 3.9 & 20.3 & 22.4 & 24.5 & 0.513 \\
\hline 99 & 22 & 14 & 59.56 & 0 & 0 & 28.3 & 20.4 & 21.7 & 24.3 & -2.000 \\
\hline 103 & 22 & 14 & 59.28 & -0 & 0 & 57.9 & 20.7 & 23.0 & 24.2 & 1.159 \\
\hline 105 & 22 & 14 & 59.18 & -0 & 1 & 12.6 & 20.8 & 22.9 & 23.9 & 1.369 \\
\hline 127 & 22 & 14 & 56.67 & -0 & 0 & 44.4 & 20.2 & 22.3 & 24.1 & 0.695 \\
\hline 142 & 22 & 14 & 54.51 & -0 & 0 & 21.7 & 20.9 & 22.6 & 24.4 & -2.000 \\
\hline 155 & 22 & 14 & 52.57 & -0 & 0 & 21.9 & 20.5 & 22.8 & 24.4 & 0.665 \\
\hline 164 & 22 & 14 & 51.53 & 0 & 0 & 41.7 & 20.1 & 22.4 & 24.0 & 0.907 \\
\hline 167 & 22 & 14 & 51.34 & 0 & 0 & 24.0 & 20.6 & 22.2 & 23.7 & 0.000 \\
\hline 176 & 22 & 14 & 50.25 & 0 & 0 & 41.2 & 20.3 & 22.2 & 23.8 & 0.767 \\
\hline 178 & 22 & 14 & 50.06 & 0 & 0 & 32.2 & 20.2 & 22.0 & 23.0 & 0.207 \\
\hline 181 & 22 & 14 & 49.75 & 0 & 0 & 32.5 & 20.2 & 22.3 & 23.6 & 0.209 \\
\hline 182 & 22 & 14 & 49.56 & -0 & 0 & 38.6 & 20.2 & 22.5 & 23.5 & 0.214 \\
\hline 189 & 22 & 14 & 48.14 & 0 & 0 & 21.8 & 20.9 & 22.8 & 24.1 & 0.275 \\
\hline 195 & 22 & 15 & 9.90 & -0 & 1 & 11.5 & 21.3 & 23.0 & 24.9 & 1.091 \\
\hline 196 & 22 & 14 & 59.19 & -0 & 1 & 10.4 & 22.8 & 22.7 & -27.9 & -2.000 \\
\hline 198 & 22 & 15 & 0.54 & -0 & 0 & 56.1 & 20.0 & 23.0 & 24.6 & 0.000 \\
\hline 199 & 22 & 15 & 6.01 & -0 & 0 & 54.0 & 20.3 & 22.6 & 25.4 & -1.000 \\
\hline 200 & 22 & 14 & 54.69 & -0 & 0 & 51.0 & 20.3 & 22.4 & 24.6 & -1.000 \\
\hline 201 & 22 & 15 & 6.14 & -0 & 0 & 43.4 & 20.9 & 22.8 & 24.6 & -1.000 \\
\hline 202 & 22 & 15 & 5.03 & -0 & 0 & 33.7 & 20.9 & 22.7 & 24.5 & -1.000 \\
\hline 203 & 22 & 15 & 1.62 & -0 & 0 & 32.0 & 21.0 & 22.6 & -27.9 & -2.000 \\
\hline 204 & 22 & 14 & 57.84 & -0 & 0 & 29.5 & 21.5 & 23.0 & 24.7 & 0.709 \\
\hline 205 & 22 & 14 & 56.58 & -0 & 0 & 25.7 & 20.0 & 21.9 & 26.2 & -2.000 \\
\hline 206 & 22 & 14 & 53.47 & -0 & 0 & 21.5 & 20.5 & 22.9 & 24.8 & -1.000 \\
\hline 207 & 22 & 15 & 8.36 & -0 & 0 & 18.9 & 20.5 & 22.5 & 24.9 & 0.663 \\
\hline 208 & 22 & 14 & 50.10 & -0 & 0 & 17.7 & -21.8 & 22.9 & 24.9 & -1.000 \\
\hline 209 & 22 & 14 & 49.80 & -0 & 0 & 10.8 & 20.9 & 22.0 & -27.0 & -2.000 \\
\hline 210 & 22 & 15 & 3.58 & -0 & 0 & 3.4 & 20.3 & 22.4 & 25.0 & -1.000 \\
\hline 211 & 22 & 14 & 48.82 & 0 & 0 & 1.7 & 21.8 & 23.0 & 24.9 & 0.657 \\
\hline 212 & 22 & 14 & 47.97 & 0 & 0 & 2.7 & -24.8 & 22.9 & 24.8 & -1.000 \\
\hline 213 & 22 & 14 & 49.52 & 0 & 0 & 11.8 & 20.8 & 22.2 & 24.9 & -2.000 \\
\hline 214 & 22 & 15 & 7.97 & 0 & 0 & 11.6 & 21.7 & 22.8 & 25.2 & -1.000 \\
\hline 215 & 22 & 14 & 50.13 & 0 & 0 & 13.7 & 22.3 & 22.8 & 25.7 & -2.000 \\
\hline 216 & 22 & 14 & 56.27 & 0 & 0 & 21.8 & 20.8 & 22.5 & 24.4 & 0.416 \\
\hline 217 & 22 & 14 & 59.71 & 0 & 0 & 47.2 & 21.0 & 22.9 & 25.9 & -2.000 \\
\hline 218 & 22 & 14 & 56.53 & 0 & 0 & 57.9 & 22.9 & 22.6 & 24.9 & 0.000 \\
\hline 29 & 22 & 15 & 7.57 & 0 & 0 & 57.2 & 22.0 & 23.9 & 24.3 & 0.000 \\
\hline 31 & 22 & 15 & 7.40 & -0 & 0 & 15.7 & -22.9 & 23.6 & 24.2 & 1.312 \\
\hline 38 & 22 & 15 & 6.81 & 0 & 0 & 25.1 & 20.8 & 23.0 & 24.2 & 1.208 \\
\hline 56 & 22 & 15 & 5.37 & 0 & 0 & 38.0 & 21.7 & 23.0 & 24.5 & 0.318 \\
\hline 58 & 22 & 15 & 5.08 & 0 & 0 & 28.7 & 20.9 & 23.3 & 24.1 & 0.000 \\
\hline 76 & 22 & 15 & 2.14 & 0 & 0 & 30.6 & 21.0 & 23.2 & 24.0 & 0.000 \\
\hline 97 & 22 & 14 & 59.78 & -0 & 0 & 30.0 & 20.1 & 23.1 & 24.4 & 0.000 \\
\hline 113 & 22 & 14 & 58.34 & -0 & 0 & 40.3 & -21.8 & 23.5 & 24.0 & 0.708 \\
\hline 115 & 22 & 14 & 58.31 & -0 & 1 & 7.6 & 22.1 & 23.6 & 24.5 & 0.709 \\
\hline 119 & 22 & 14 & 57.89 & 0 & 0 & 32.7 & 21.3 & 23.6 & 24.3 & 0.000 \\
\hline 121 & 22 & 14 & 57.47 & 0 & 0 & 45.0 & 21.7 & 23.0 & 24.2 & 0.215 \\
\hline 128 & 22 & 14 & 56.32 & 0 & 0 & 57.5 & 21.3 & 23.1 & 23.8 & 1.510 \\
\hline
\end{tabular}


TABLE 1(b). (continued)

\begin{tabular}{|c|c|c|c|c|c|c|c|c|c|c|}
\hline \multirow{2}{*}{$\frac{\#}{132}$} & \multicolumn{3}{|c|}{ R.A. (1950) } & \multicolumn{3}{|c|}{ Dec. (1950) } & \multirow{2}{*}{$\frac{K}{22.3}$} & \multirow{2}{*}{$\frac{I}{23.4}$} & \multirow{2}{*}{$\frac{B}{24.2}$} & \multirow{2}{*}{$\frac{z}{1.010}$} \\
\hline & 22 & 14 & 55.48 & 0 & 0 & 18.4 & & & & \\
\hline 133 & 22 & 14 & 55.06 & -0 & 0 & 48.6 & 22.2 & 23.1 & 24.5 & 0.543 \\
\hline 139 & 22 & 14 & 54.79 & -0 & 0 & 9.3 & -23.1 & 23.2 & 24.1 & 0.872 \\
\hline 163 & 22 & 14 & 51.72 & 0 & 0 & 9.4 & 20.8 & 23.5 & 24.1 & 0.000 \\
\hline 192 & 22 & 14 & 47.67 & 0 & 0 & 19.3 & 20.2 & 23.1 & 24.0 & 0.000 \\
\hline 193 & 22 & 14 & 47.63 & 0 & 0 & 25.8 & 20.7 & 23.1 & 23.9 & 1.678 \\
\hline
\end{tabular}

Notes to TABLE $1 b$

The SSA22 sample selected to $K=20, I=23$, and $B=24.5$. Objects with redshifts designated -1.000 were not observed; those with redshifts designate -2.000 were identified as stars.

two of the authors independently. Where a redshift was considered secure (i.e., definitely identified by both observers) it was entered into the catalog of Tables $1 \mathrm{a}$ and $1 \mathrm{~b}$ and generally excluded from future mask design unless there were no further unidentified objects at this position on the E-W slit axis of the mask. However, dubious or unidentified objects were included in additional masks until such time as the summed spectrum produced a reliable identification. The longest exposure on a single object was six hours. Objects which still do not have a robust identification are marked by zeros in the catalog of Tables $1 \mathrm{a}$ and $1 \mathrm{~b}$, while stars are marked with -2 and unobserved objects with -1 . For the
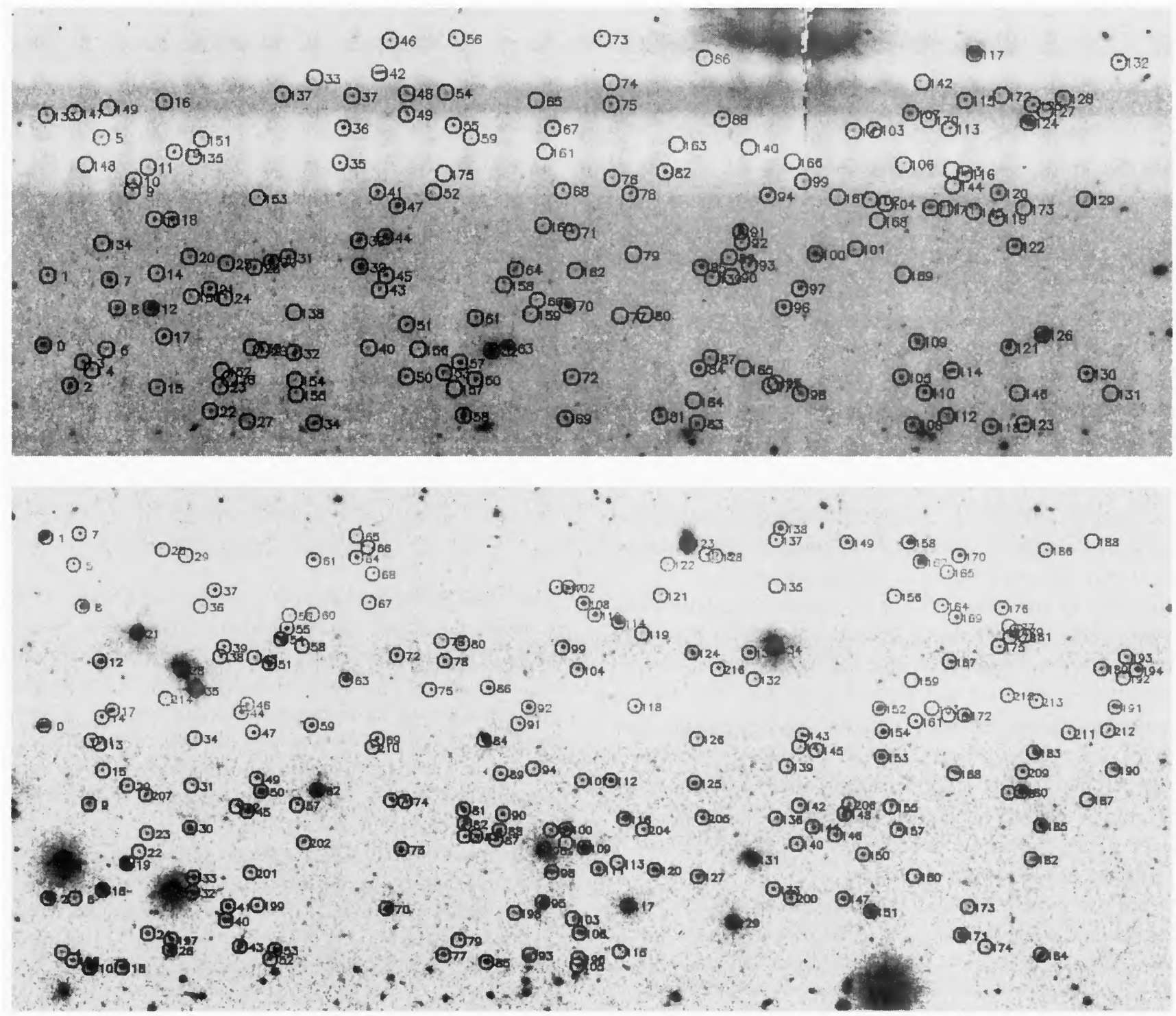

FIG. 1. (a) $I$-band finding chart for the objects in Table 1a. Each object is circled and labelled with the running object number given in column 1 of the table. (b) The corresponding $I$-band finding chart for Table $1 \mathrm{~b}$. 

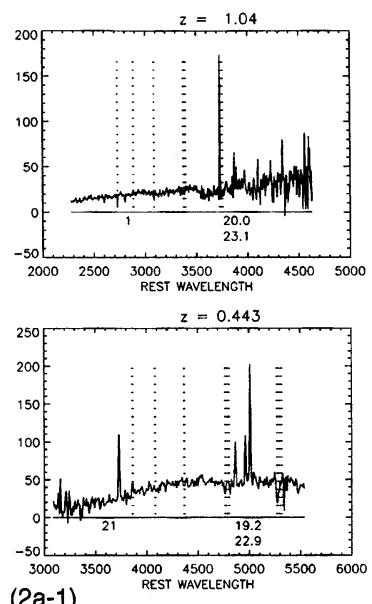

(2a-1)
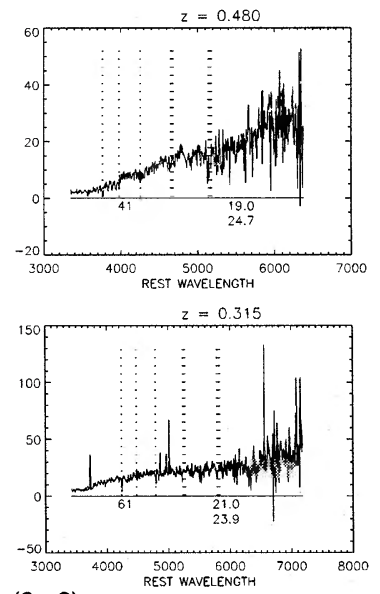

(2a-2)
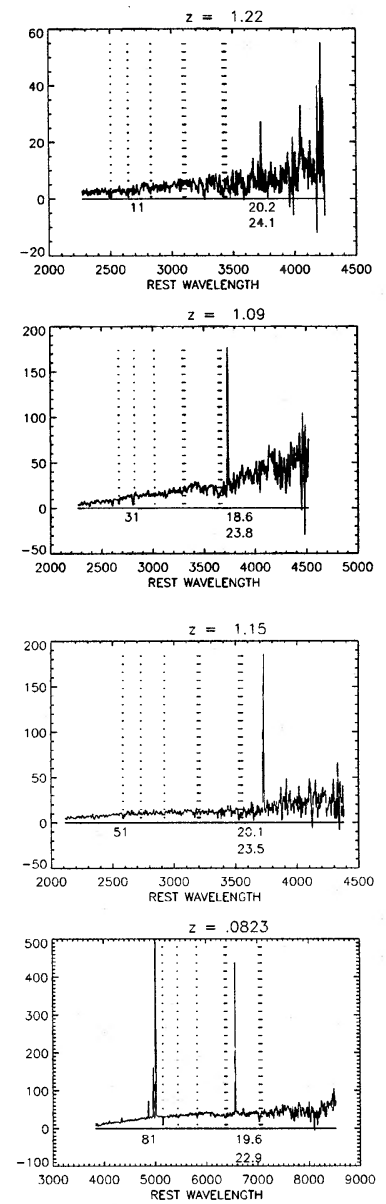
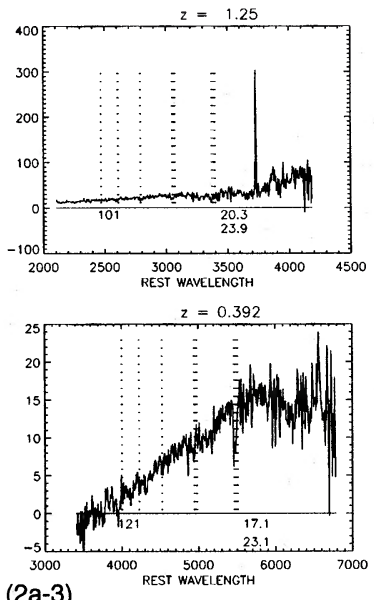

(2a-3)

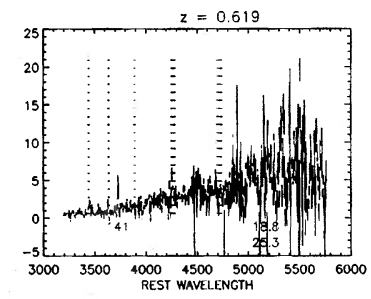

(2a-4)
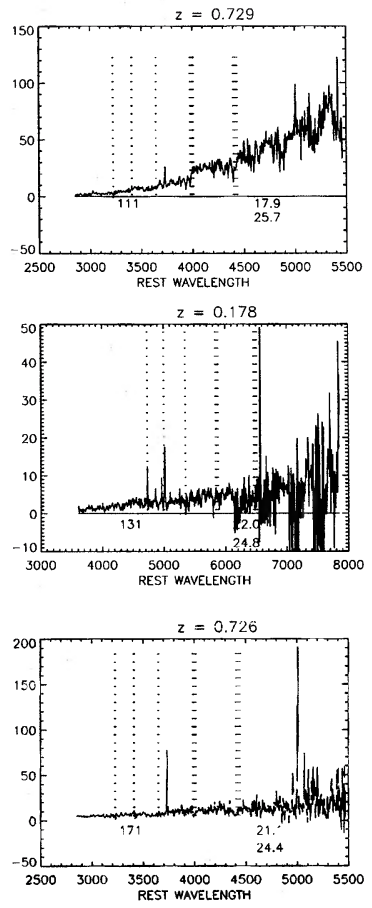

FIG. 2. (a) Spectra of every 10th object in Table 1a, excluding stars and unidentified objects. The spectra are shown in the rest frame corresponding to the redshift given at the top of each panel, and are approximately $f_{\nu}$ in arbitrary units. The shaded regions mark the positions of the stronger night sky lines where residuals may be present in the spectra, and the positions of the atmospheric bands. The $K$ and $B$ magnitudes are shown at the lower right, and the identifying number from Table 1(a) (column 1) at the lower left.

completeness analysis, we consider unidentified objects and unobserved objects to be equivalent, but for some statistical tests it may be appropriate to distinguish them.

Because of the large number of objects, it is not practicable to show all the spectra. Instead we show in Figs. 2(a) and 2(b) every 10th object, excluding stars and unobserved objects (designated -2 and -1 in the catalog). The spectra are presented in the rest frame with the shaded regions showing the positions of strong sky features (the atmospheric bands and the stronger night sky lines). The redshift is shown at the top of the plot, the number of the object in Tables 1a or $1 \mathrm{~b}$ and Figs. 1(a) or 1(b) at the lower left, and the $K$ and $B$ magnitudes at the lower right.

Finally, we show in Fig. 3 the completeness as a function of magnitude in each color, as well as the number of objects in the catalog. For the SSA 13 field we have included objects only to $B<24$ because a relatively large number of objects with $B=24-24.5$ have yet to be observed in this field. The samples are reasonably complete to $K=19.5, I=22.5$, and $B=24.5$.

\section{THE $K$-BAND SAMPLE}

\section{$3.1 \mathrm{~K}-\mathrm{z}$ Relation}

We have combined the present data for $K=18-20$ with the much larger area samples to $K<18$ given in Songaila et al. (1994). The Songaila et al. sample ranges in area from $1.54 \mathrm{deg}^{2}$ at $K<14.5$ to $89 \operatorname{arcmin}^{2}$ at $K<18$ and is nearly fully complete at these magnitudes. The combined sample contains 416 objects, of which 31 are stars and 346 are galaxies with well determined redshifts. From Fig. 3 it can be seen that the sample is $77 \%$ complete at $K=19-19.5$, but only $57 \%$ complete at $K=19.5-20$.

We show the redshift-magnitude relation in Fig. 4, with filled triangles giving the Songaila et al. sample and filled squares showing data from the present catalog. The diagram has been extended to $K=21$, but beyond $K=20$ (the vertical dashed line) there is, of course, a strong color bias in the sample since it contains only objects with $I<23$ or $B<24.5$. Unidentified objects are not shown in this plot. Other available samples are also shown in Fig. 4-the open symbols show the Mobasher et al. (1986) and Glazebrook et al. 

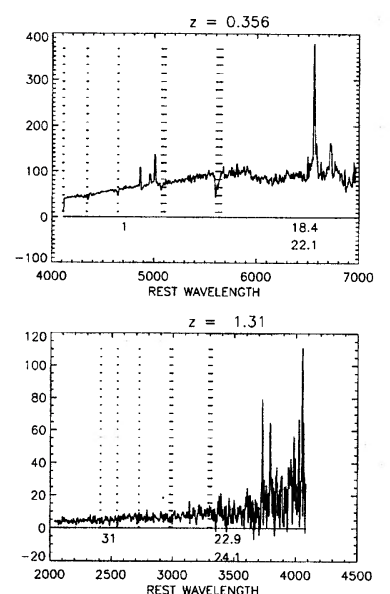

(2b-1)
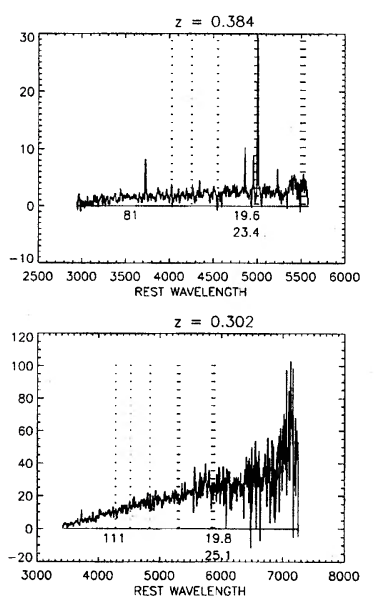

$(2 b-2)$
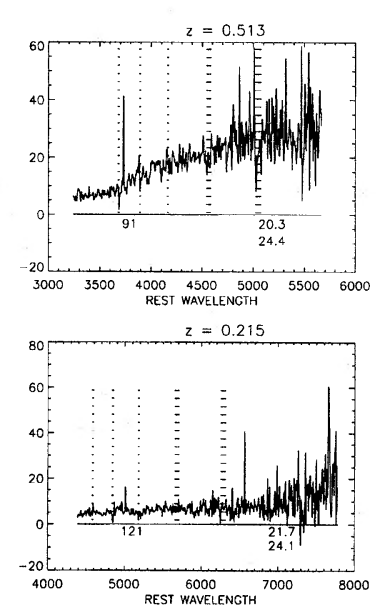
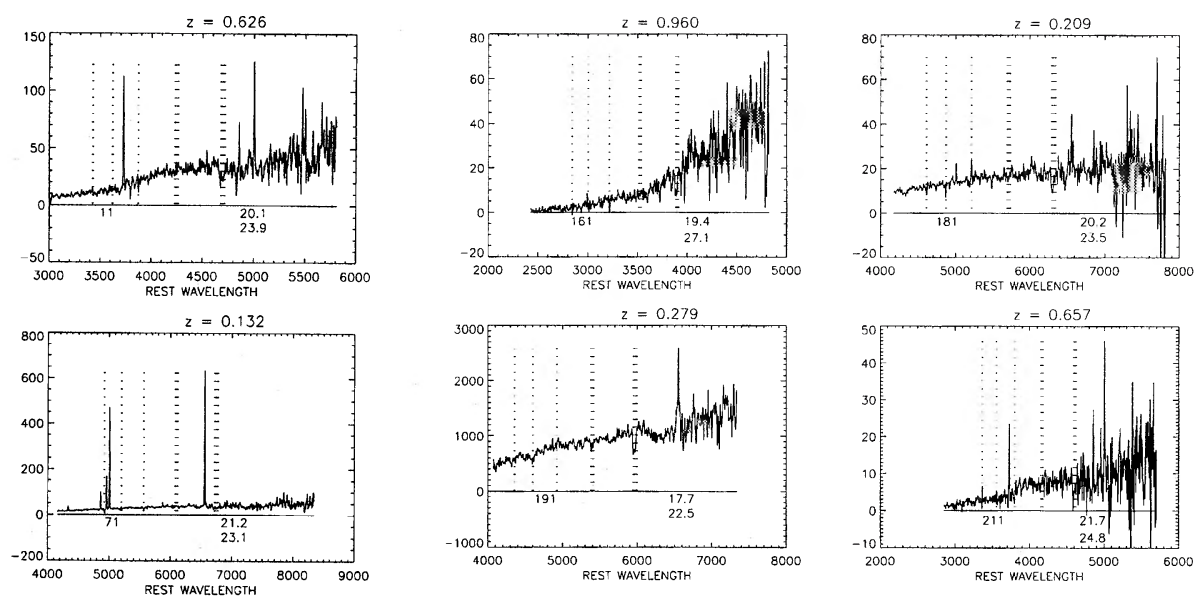

(2b-3)

FIG. 2(b). Spectra of every 10th object in Table 1(b) excluding stars and unidentified objects. The spectra are shown in the rest frame corresponding to the redshift given at the top of each panel, and are approximately $f_{\nu}$ in arbitrary units. The shaded regions mark the positions of the stronger night sky lines where residuals may be present in the spectra, and the positions of the atmospheric bands. The $K$ and $B$ magnitudes are shown at the lower right, and the identifying number from Table 1(b) (column 1) at the lower left.

The various Hawaii samples use apertures of sufficiently large angular diameter so that small average corrections may be applied to obtain approximate total magnitudes, whereas Mobasher et al. (1986) used corrected isophotal magnitudes. For comparison purposes we have therefore used the Glazebrook et al. (1995b) metric magnitudes measured in a $40 h_{50}^{-1}$ kpc aperture (where $h_{50}=H_{0} / 50 \mathrm{~km} \mathrm{sec}^{-1} \mathrm{Mpc}^{-1}$ ), which are closer to the total magnitudes used here and by Mobasher et al., and we have cut off their sample at $K=17$. Because of the different methodologies, there may be internal dispersions as large as 0.2 to $0.3 \mathrm{mag}$ (e.g., Cowie et al. 1994, hereafter Paper I), and smaller systematic offsets between the samples.

Figure 4 can be used to see many of the conclusions of this section in a semi-quantitative fashion. Here we have plotted two curves, the lower one for a $0.1 L_{*}$ galaxy and the upper one for a $3 L_{*}$ galaxy, for $M_{K *}=-25.0\left(+5 \log _{10} h_{50}\right)$ where we have computed $K$ versus $z$ using $q_{0}=0.5$ and the $K$-correction for an $\mathrm{Sb}$ galaxy given in Paper I and overlaid these on the $K-z$ distribution of objects from both the present sample and the Glazebrook et al. and Mobasher et al. samples. These extrema should roughly bound the distribution of redshifts, given an $\alpha=-1$ Schechter function, as indeed they do at lower redshifts. However, at fainter magni-

FIG. 3. Completeness of the sample in each color as a function of magnitude. The completeness is defined here as the ratio of the total number of identified objects (stars and galaxies) to the total number of objects in the catalog (whether observed or not). Data from Table 1a (SSA 13 field) are only included for $I<22.5$ and $B<24$. The total number of objects in each color sample is shown at the lower left. 


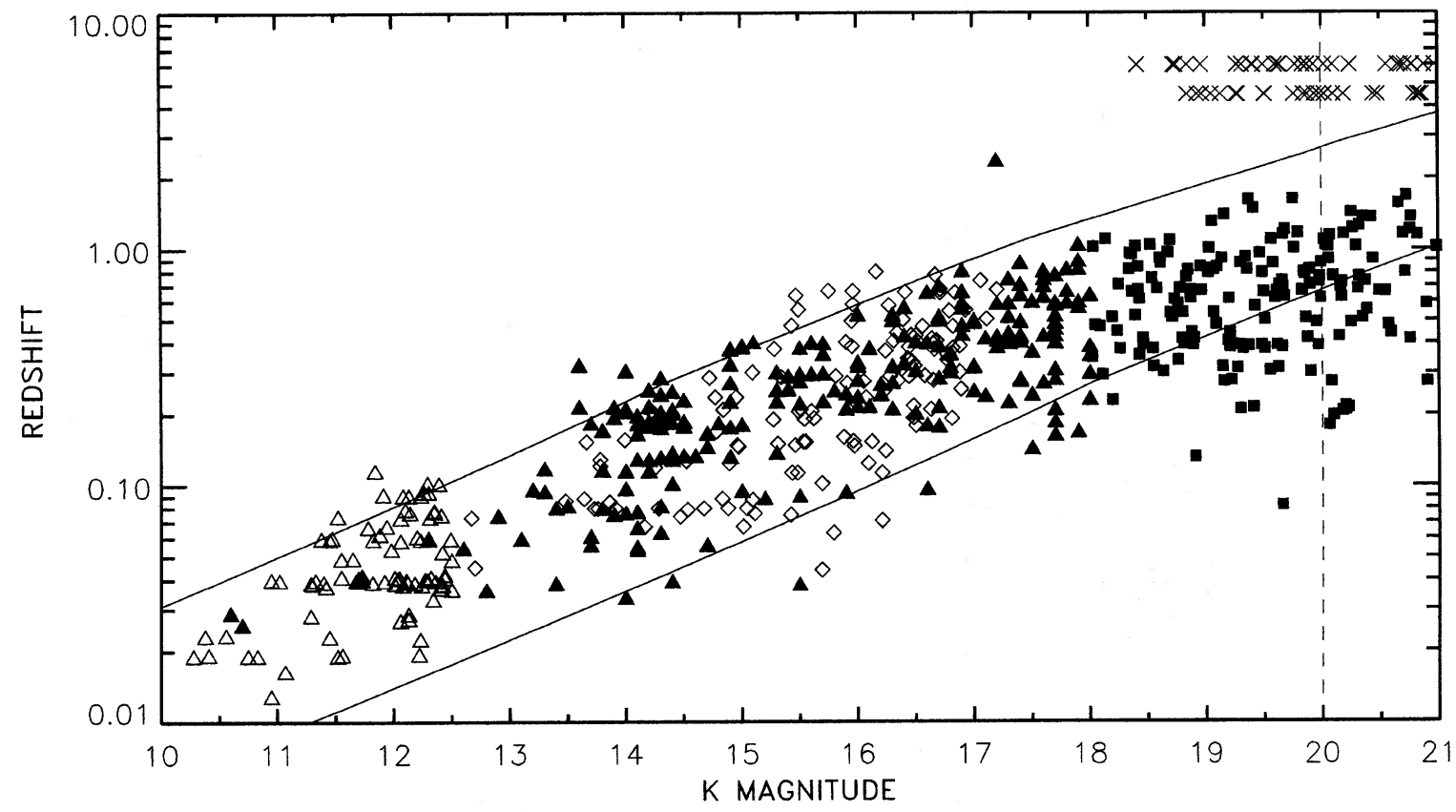

FIG. 4. Redshift vs $K$ magnitude for the present sample at $K>18$ (filled squares). At $K \leqslant 18$ we show the much larger Songaila et al. (1994) sample (filled triangles) which contains the present areas as a subsample. Open triangles show the data of Mobasher et $_{\text {al }}$. (1986) and open diamonds the $40 h_{50}^{-1} \mathrm{kpc}$ metric magnitudes of Glazebrook et al. (1995b). We have also shown the unidentified or unobserved objects in Tables la and 1b as crosses at an arbitrary redshift for each field. The solid lines show the apparent $K$ magnitude for an Sb galaxy with $L=3 L_{*}$ (upper) and $L=0.1 L_{*}$ (lower), where we take $M_{K *}=-25.0+5 \log _{10} h_{50}$.

tudes the redshifts begin to fall systematically low. This may be more clearly seen in Fig. 5, where we show the median redshift versus $K$ magnitude for the present sample and those of Glazebrook et al. and Mobasher et al.. The $1 \sigma$ error bars are calculated in a highly conservative fashion by using the median sign method, allowing for the possibility that unidentified or unobserved objects may be at either high or low redshift. At faint magnitudes the data points lie between the predictions of a no luminosity evolution model computed here for a Schechter function with $M_{K *}=-25.0$ $+5 \log _{10} h_{50}, \alpha=-1$, and $q_{0}=0.5$ and $q_{0}=0.02$ (solid and dashed lines, respectively) and the lower values predicted by a strong merger model such as those of Rocca-Volmerange \& Guiderdoni (1990), Broadhurst et al. (1992), and Carlberg (1992) (whose model's predictions are shown as a dotted line on the figure), but are well below even a mild passive luminosity evolution model, illustrated here by the dash-dot line. The $K$ luminosity per galaxy would therefore appear to be invariant, or even slightly falling, at higher redshifts.

\subsection{The Evolution of the K-band Luminosity Function}

For each galaxy we have determined a rest-frame absolute $K$ magnitude using the $K$-correction for an $\mathrm{Sb}$ galaxy given in Paper I as

$$
M_{K}=m_{K}-5 \log \left(\frac{d_{L}}{10 \mathrm{pc}}\right)-K(z),
$$

where $d_{L}$ is the luminosity distance and $m_{K}$ the apparent $K$ magnitude. Between $z=0$ and 1 , the use of a typeindependent $K(z)$ correction is justified for the $K$ band and introduces at most a $0.1 \mathrm{mag}$ error for an elliptical and 0.2 mag for an Im, errors smaller than or comparable to the photometric uncertainties of $\sim 0.2 \mathrm{mag}$ in the catalogs. This type invariance of the $K$ correction greatly simplifies the analysis.

The sample was next sorted by absolute magnitude from the most negative (most luminous) to the most positive values, and the observable comoving volumes between redshifts $z_{1}$ and $z_{2}$ computed for each galaxy as

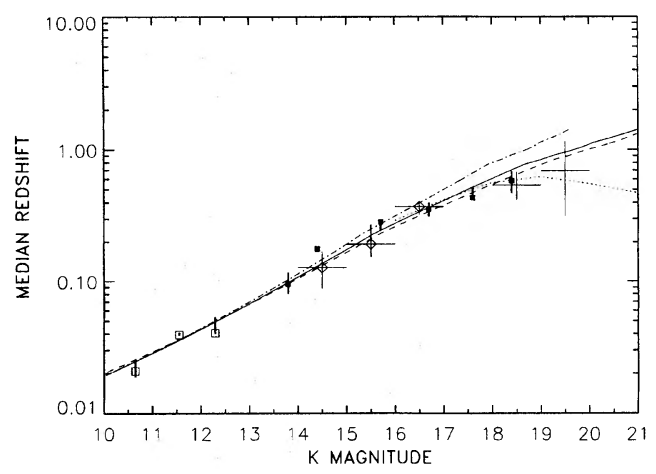

FIG. 5. Median redshift as a function of $K$ magnitude. The open squares are from Mobasher et al. (1986), the filled squares from Songaila et al. (1994), the open diamonds from Glazebrook et al. (1995b), and the pluses from the present work. In each bin the error bars are $\pm 1 \sigma$ based on the median sign method, allowing unidentified or unobserved objects to be at either high or low redshift. The solid $\left(q_{0}=0.5\right)$ and dashed $\left(q_{0}=0.02\right)$ lines show the predictions for a non-evolving luminosity function with $M_{K *}=-25.0$ $+5 \log _{10} h_{50}$ and $\alpha=-1.25$. The dash-dot line shows a model with mild luminosity evolution while the dotted line shows the prediction of a merger model Carlberg 1992). 


$$
V_{j}=\frac{c}{H_{0}} \sum_{i} A_{i} \int_{z_{l i}}^{z_{u i}} \frac{d_{l}^{2} d z}{(1+z)^{3}\left(1+2 q_{0} z\right)^{0.5}},
$$

where $z_{l i}$ is the maximum of $z_{1}$ and the minimum redshift at which object $j$ lies in the $i$ th magnitude range, and $z_{u i}$ is the minimum of $z_{2}$ and the maximum redshift at which object $j$ lies in the $i$ th magnitude range. $A_{i}$ is the observed area in the $i$ th magnitude range. We then constructed the cumulative $K$-band luminosity per unit comoving volume to an absolute magnitude $M_{K}$ corresponding to object $k$ as

$$
\lambda_{K} \equiv \lambda\left(M_{K}\right)=\sum_{j=1}^{k} \frac{L_{j}}{V_{j}},
$$

which we shall refer to as the cumulative luminosity density, and the cumulative number per comoving volume

$$
n\left(M_{K}\right)=\sum_{j=1}^{k} \frac{1}{V_{j}} .
$$

Here $L_{j}$ is the $K$-band luminosity of object $j$ in solar units, with $M_{K \odot}=3.41$.

This cumulative expression allows us to display the form of the luminosity function without binning or parametric fits, and the smoothness of the function may be used to visually estimate the degree of uncertainty. The principal demerit of this approach is that the errors are no longer independent.

In Fig. 6(a) we plot the cumulative rest-frame $K$-band luminosity density versus $M_{K}$ computed for the redshift ranges $[0,0.3]$ (crosses), $[0.3,1]$ (boxes), and $[1,1.6]$ (diamonds) for $q_{0}=0.02$ and 0.5 ; while in Fig. 6(b) we show the corresponding number of objects in each magnitude bin. The median redshift is 0.18 in the lowest redshift interval, 0.51 in the $z=[0.3,1]$ interval, and 1.10 in the $z=[1,1.6]$ range. The most striking point is that the faint-end asymptotic luminosity density has risen by a factor of 1.4 to 1.6 (depending on the assumed $q_{0}$ ) between the two lower redshift ranges, a point to which we shall return in the next section. Here we concentrate on the shape of the luminosity function and, in order to visually compare the shapes of the distributions in the two redshift intervals, we have renormalized the $z=[0.3,1]$ distribution to have the same light density at $M_{K}=-21+5 \log _{10} h_{50}$ (shown by the solid line). It can then be seen that the solid line is shifted to slightly fainter magnitudes for $q_{0}=0.5$ and is invariant for $q_{0}=0.02$. Because the highest redshift bin extends only over a small absolute magnitude interval, it is not possible to compare the shape in this way, but it is also consistent with an invariant or declining $M_{K *}$. In particular, galaxies with $M_{K}<-26+5 \log _{10} h_{50}$ have $K<18.5$ at $z=1.6$ and, therefore, lie within the essentially completely identified sample; it must be, then, that the deficiency of luminous objects at these high redshifts is not a selection effect.

We have formally fitted a Schechter function to the cumulative number distribution in the two lower redshift bins using the Kolmogorov-Smirnov test to determine $68 \%$ confidence contours. These are shown in Fig. 7 for the two parameters, $\alpha$ and $M_{K *}$. The best fit to the low-redshift interval gives $\alpha=-1.2$ and $M_{K *}=-25.0+5 \log _{10} h_{50}$. At higher redshift for $q_{0}=0.5$ the best fit is $\alpha=-1.3$,
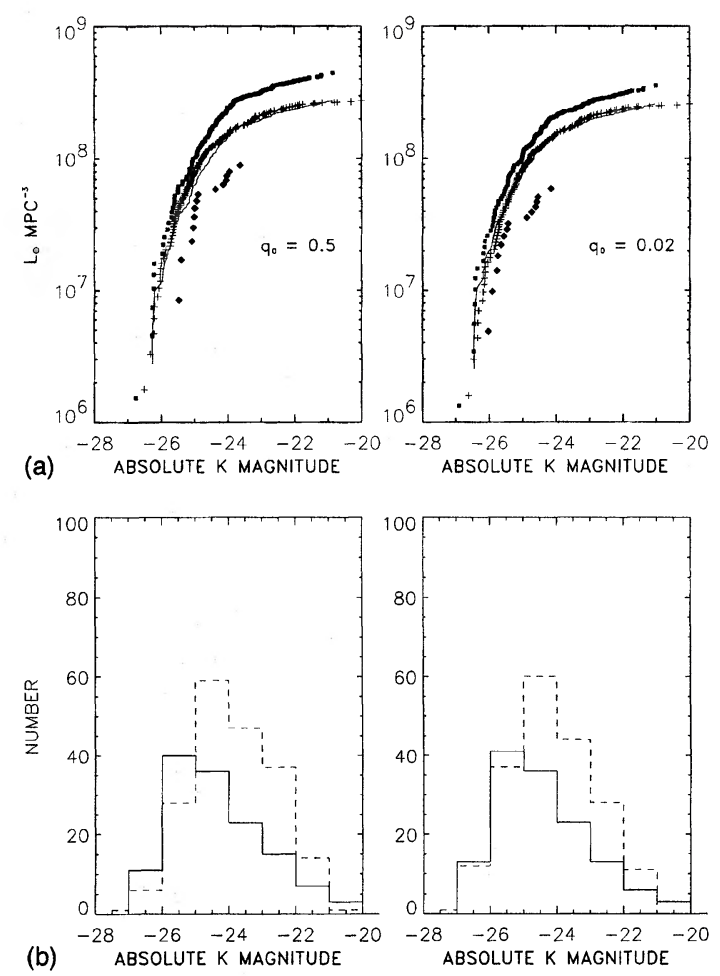

FIG. 6. (a) The cumulative comoving rest-frame $K$-band luminosity density from galaxies more luminous than a given rest-frame absolute $K$ magnitude. The pluses correspond to the redshift ranges $z=[0,0.3]$, filled squares to $z=[0.3,1]$, and filled diamonds to $z=[1,1.6]$. The left panel shows $q_{0}=0.5$, the right, $q_{0}=0.02$. The solid line shows the $z=[0.3,1]$ curve renormalized to match the local luminosity density. Note that the asymptotic luminosity density has risen by roughly a factor of 1.4 to 1.6 (depending on $q_{0}$ ) between the first two redshift intervals (pluses and filled squares). The $K$ magnitude cutoff for the sample does not allow similar extrapolation for the $z=[1,1.6]$ interval, but the marked divergence of its curve at luminosities brighter than $M_{K} \approx-25$ and the change in its shape with respect to the cumulative luminosity density functions of the lower two redshift intervals may be noted. (b) The corresponding histogram of the number of objects in each absolute magnitude bin. The solid line is for the $z=[0,0.3]$ redshift interval and the dashed is for $z=[0.3,1]$. The left panel is for $q_{0}=0.5$, the right is for $q_{0}=0.02$.

$M_{K *}=-25.0+5 \log _{10} h_{50}$, while for $q_{0}=0.02$ it is $\alpha=-1.4$, $M_{K *}=-25.4+5 \log _{10} h_{50}$. Given the statistical and photometric uncertainties, the results are best described as indicating an invariant, or, depending on the assumed $q_{0}$, a very slightly decreasing, galaxy luminosity.

The present results agree well with those of Mobasher et al. (1993) who find $\alpha=-1$ and $M_{K *}=-25.0 \pm 0.3$ $+5 \log _{10} h_{50}$ in their low-redshift bright sample. However, Glazebrook et al. (1995b) find $M_{K *}=-24.25 \pm 0.13$ $+5 \log _{10} h_{50}$ at $z=0.0-0.4$ and $M_{K *}=-24.91 \pm 0.15$ $+5 \log _{10} h_{50}$ at $z=0.4-0.8$, where they force fit in both cases to an $\alpha=-1$ power law. At the higher redshift this agrees well with the present analysis but the $M_{K *}$ is considerably fainter at low redshift than the value determined here even when allowance is made for possible differences $(\sim 0.3$ mag) between the $40 h_{50}^{-1} \mathrm{kpc}$ metric magnitudes used by Glazebrook et al. and the present magnitudes. This led Glazebrook et al. to conclude that there was positive luminosity evolution with increasing $z$, in disagreement with our result. 

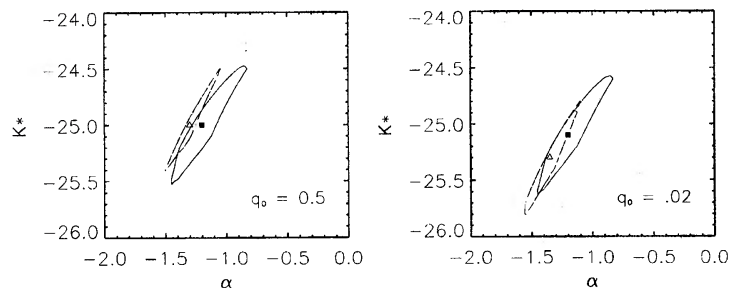

FIG. 7. $1 \sigma$ error contours for $K_{*}$ and $\alpha$ in a Schechter function fit to the cumulative number distribution of the data at $z=[0,0.3]$ (solid) and $z=[0.3,1]$ (dashed). The filled square shows the best fit at low redshift and the open triangle the best fit at high redshift, and within the statistical and photometric uncertainties these describe an invariant, or possibly even declining (depending on $q_{0}$ ) $K$-band luminosity across the spanned redshift interval.

As Glazebrook et al. note, this difference may result from the fluctuations inherent in small samples; the low number of super- $L_{*}$ galaxies can result in biases in $M_{K *}$. Our $z=[0,0.3]$ bin contains 138 galaxies, the Mobasher et al. sample 95 galaxies, and the Glazebrook et al. $z<0.4$ bin 98 galaxies.

In order to understand the disagreement better, we have therefore performed the same analysis on the combined data sets. With 296 galaxies in the lower redshift $z=[0,0.3]$ bin, we find a best fit of $M_{K *}=-24.8+5 \log _{10} h_{50}$ and $\alpha=-1.1$. In the higher redshift bin with 232 galaxies, we find $M_{K *}=-24.9+5 \log _{10} h_{50}$ and $\alpha=-1.3$ for $q_{0}=0.5$ and $M_{K *}=-25.1+5 \log _{10} h_{50}, \alpha=-1.3$ for $q_{0}=0.02$. The results are again consistent with shape invariance $\left(q_{0}=0.02\right)$ or a very slight dimming with increasing redshift $\left(q_{0}=0.5\right)$.

An alternative presentation is given in Fig. 8, where we have directly computed the comoving luminosity functions in four redshift intervals using the $1 / V$ method for $q_{0}=0.5$. Poisson errors are shown, corresponding to the number of objects in each magnitude bin. Where no objects were detected in a bin, the $1 \sigma$ upper limit is shown with a downward pointing arrow. Once again we have shown the Hawaii data only (solid squares) and the combined data (open squares). The fits (solid line for the Hawaii data, dashed line for all data) are shown for a $M_{K *}=-25.0+5 \log _{10} h_{50}, \alpha=-1.25$ Schechter function, which provides an acceptable fit in al four redshift intervals (irrespective of which data set is used) with a suitable choice of the normalization, $\phi_{*}$. The dotted line shows a reference value of $\phi_{*}=10^{-3} h_{50}^{3} \mathrm{Mpc}^{-3}$. The normalization lies at or below this value in the $z<0.1$ and $z=0.1-0.2$ bins, rises to roughly a factor of two higher in the $z=0.2-0.6 \mathrm{bin}$, and then begins to drop again at $z=0.6$ -1.0 .

\subsection{The Evolution of Colors and Emission Line Strengths in the K Sample}

The $(I-K)$ versus $K$ diagram for the present sample $(K$ or $I$-selected) is shown in Fig. 9(a) with objects marked as stars (asterisks), identified galaxies at $z<1$ (filled boxes), galaxies at $z>1$ (boxes surrounded by open boxes), and unidentified objects (pluses). The error bars are $\pm 1 \sigma$ errors in $I$ only. The figure demonstrates that the incompleteness in the $K$ sample is primarily in objects with redder $(I-K)$ colors.
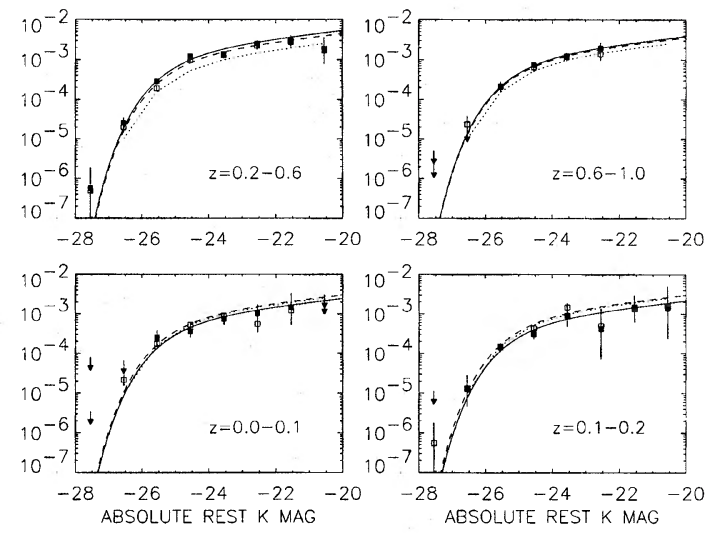

FIG. 8, $K$-band luminosity function as a function of redshift slice. The filled squares show only the Hawaii data (with downward pointing arrowheads indicating $1 \sigma$ upper limits in bins where no objects were detected), while the open squares also include the Mobasher et al. (1993) and Glazebrook et al. (1995b) data. The solid line shows the best fit Schechter function with $M_{K *}=-25.0, \alpha=-1.25$ (Hawaii data: solid; all data: dashed) while the dotted line shows the function for $\phi_{*}=10^{-3} \mathrm{Mpc}^{-3}$.

However, as has been previously emphasized in Cowie $e t$ al. $(1994,1995 b)$, blue objects dominate the $K$ counts and many $z>1$ galaxies are contained in the bluer objects near $K=20$. There are also relatively few objects (less than eleven in this sample) which can have colors redder than an Sb galaxy at $z>1.5(I-K \geqslant 4.5)$ even though an $M_{K *}=-25.0 \mathrm{Sb}$ galaxy would have $K=19.5$ at $z=1.5$.

This point is further emphasized in Fig. 9(b) where we show the $(I-K)$ colors of a $K<22.5$ sample centered on the deep HST I-band images of Cowie et al. (1995b) which lie at the centers of the spectroscopic strips. In these areas very much deeper $K$-band images $(1 \sigma K=24.5$ in SSA 22 and $1 \sigma$ $K=23.5$ in SSA 13) have been obtained with the QUIRC $1024 \times 1024$ IR camera at CFHT. We show all objects in a 3 $\operatorname{arcmin}^{2}$ area with $K<22.5$ in SSA 22 and with $K<21.5$ in a $3 \operatorname{arcmin}^{2}$ area in SSA 13. Even in this extremely deep sample there are very few red objects (six with $(I-K)>4.5$ ) and the great bulk of $K$-selected objects are extremely blue in $(I-K)$.

To investigate the origin of this blueing trend within the present spectroscopic data, we can look at either the emission-line characteristics of the galaxies or their restframe ultraviolet colors. For each galaxy we measured the rest-frame equivalent width of the [O II] $3727 \AA$ emission line $(\mathrm{EW}([\mathrm{O} \mathrm{II}]))$ or, in cases where the redshifted [O II $]$ lay blueward of our observed spectral range, we used the equivalent width of $\mathrm{H} \alpha$ to determine $\mathrm{EW}([\mathrm{O} \mathrm{II}])=0.4 \mathrm{EW}(\mathrm{H} \alpha)$ (Kennicutt 1992; Songaila et al. 1994). EW([O II]) is shown in Fig. 10 as a function of the rest absolute $K$ magnitude and of redshift $z$; this is perhaps the most striking single result of the present paper.

At low redshifts (lower left panel) very few galaxies have very strong [O II] lines. Roughly speaking an EW([O II $]$ ) $=25 \AA$ separates galaxies undergoing rapid formation (mass doubling in less than $10^{10} \mathrm{yr}$ ) from those which are undergoing low rates of star formation (Kennicutt 1992), and in this panel only a small number of very low-mass galaxies fall in the fast-formation category. As we move to higher redshift, 

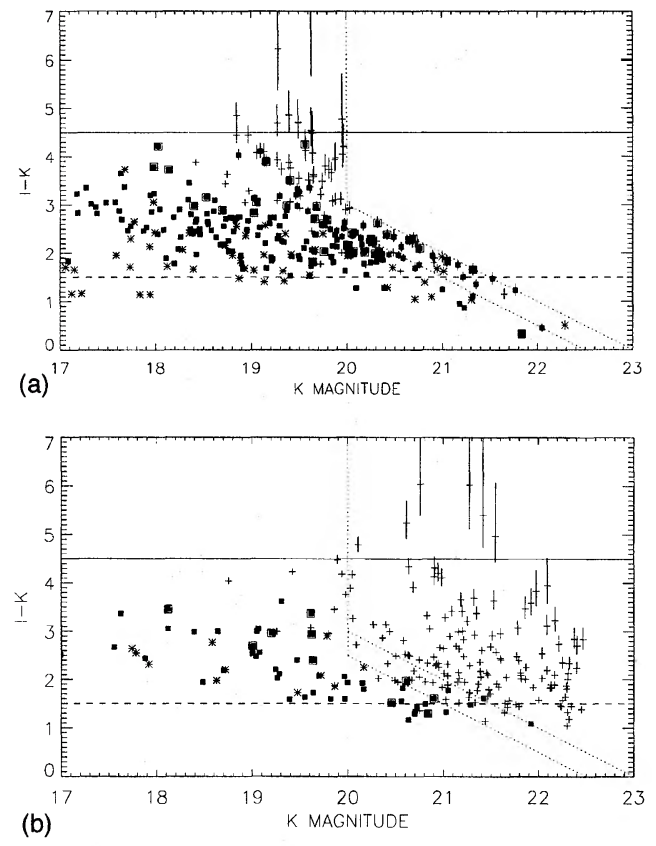

FIG. 9. (a) $(I-K)$ vs $K$ for the present sample (panel $a$ ). All objects are shown with $\pm 1 \phi$ error bars in $I$. The dotted lines show the magnitude limits of the current spectroscopic samples while the solid line shows the expected $(I-K)$ color of a $z=1.5 \mathrm{Sb}$ galaxy, and the dashed line indicates flat $f_{\nu}$. Identified objects are shown as asterisks (stars), filled squares (galaxies with $z<1$ ), and boxed filled squares (galaxies with $z>1$ ), with unidentified objects designated with pluses. (b) The corresponding $(I-K)$ vs $K$ plot for a very deep sub-sample (panel $b$ ). Although the incompleteness in the $K$-selected sample is primarily in objects with redder $(I-K)$ colors [panel a], blue objects dominate the $K$ counts, and the great bulk of $K$-selected objects are extremely blue in $(I-K)($ panel $b)$.

we see progressively more massive galaxies with rapid formation, until at $z>1$ we see $M_{K *} \sim-25$ galaxies falling in this category (Cowie et al. 1995a). Because of the bias against the spectroscopic identification of red objects the higher- $z$ panels may be missing more quiescent objects but the point here is the presence of many massive rapidly starforming objects-a type of galaxy which is not seen at the present time. This effect is clearly seen in other samples: in particular, Ellis et al. (1996) have shown that the luminosity function of strong [O II] emitting galaxies evolves rapidly with redshift whereas that of the weak [O II] emitters is nearly invariant.

We can also see the same result independently from the rest-frame ultraviolet-infrared colors, which provide an independent measure of the star-formation rate in the galaxies that is more direct than the [O II] emission (Kennicutt 1992). We have measured the rest-frame $3500 \AA A B$ magnitude by assigning an approximate type to each galaxy and using this to interpolate between the observed $B$ and $I$ magnitudes and to extrapolate from $K$ to obtain the rest-frame $21000 \AA A B$ magnitude.

The type was assigned from the galaxy's $(I-K)$ color and redshift by interpolating between the predicted colors of Coleman et al. (1980) models at that redshift. The corresponding spectral energy distribution was then used to interpolate between the $B$ and $I$ magnitudes. However, it is im- portant to emphasize that the assigned rest-frame $U$ magnitude is quite insensitive to this procedure since the interpolation is relatively small and the procedure is, of course, exact at $z=0.3$ where the observed $B$ band corresponds to the rest-frame $3500 \AA$, and at $z=1.4$, where the observed $I$ magnitude corresponds to rest-frame $3500 \AA$. To show this insensitivity we have compared the rest-frame $3500 \AA$ magnitude computed with the type-interpolation procedure to a simple logarithmic interpolation where the restframe $2500 \AA$ magnitude is obtained as

$$
(3500)_{A B}=B_{A B}+\frac{\log _{10}\left(\frac{3500(1+z)}{4500}\right)}{\log _{10}\left(\frac{8400}{4500}\right)}\left(I_{A B}-B_{A B}\right) .
$$

The deviation between these two methods of assigning the rest-frame $U$ magnitude reaches a maximum of only 0.3 mag for any galaxy, which provides a very extreme upper bound to the uncertainty in the $3500 \AA$ rest-frame magnitude introduced by the interpolation.

Figure 11 shows the comparison between the [O II] equivalent widths and the rest-frame ultraviolet-infrared colors as a function of redshift and absolute $K$ magnitude. At low redshifts almost all galaxies are quiescent in both diagnostics. At $z=0.2-0.4$, there are a number of low-mass galaxies with large $\mathrm{EW}([\mathrm{O} \mathrm{II}])$ and $(3500-K)_{A B}<1.3$ which are rapid star formers, but more massive galaxies are quiescent. Finally, at higher redshifts massive galaxies are seen in the rapid formation region. We can summarize these figures as follows. It appears that at the present time there is almost no galaxy formation, but that as recently as $z \sim 0.2$, low-mass $\left(M_{K *}=-22\right)$ galaxies were forming; progressively higher galaxy masses are seen in formation at higher redshifts, to the point where near- $L_{*}\left(M_{K *}=-24\right.$ to -25$)$ galaxies are seen undergoing rapid star formation at $z>1$.

\section{THE $B$ SAMPLE \\ $4.1 B-z$ Relation}

The redshift sample was extended to $B=24.5$ in both fields by observing the additional objects not previously included in the $K=20$ sample (Tables $1 \mathrm{a}$ and $1 \mathrm{~b}$ ). Essentially all the $B=24.5$ objects in SSA 22 were observed but in SSA 13 the sample is completely observed only to $B=24$ (Tables 1a and 1b). We have therefore defined the sample as all objects with $B \leqslant 24.5$ in SSA 22 and $B<21$ in SSA 13. The total sample comprises 203 objects and is quite complete (>85\%) even at $B=24-24.5$ (Fig. 3).

The magnitude-redshift relation for the galaxies in the sample is shown in Fig. 12, where we distinguish the SSA 13 members (filled diamonds) from those in SSA 22 (filled squares). Unidentified objects are shown as diagonal crosses at a nominal redshift of 1.75 for SSA 22 and as upright crosses (pluses) at a nominal redshift of 1.85 for SSA 13. We also show as the large pluses the median redshifts for objects in 0.5 mag bins with $\pm 1 \sigma$ errors computed using the median sign method, again placing unidentified objects above or below the median to estimate the maximum error. For comparison we also show (solid line) the predicted median redshift- 

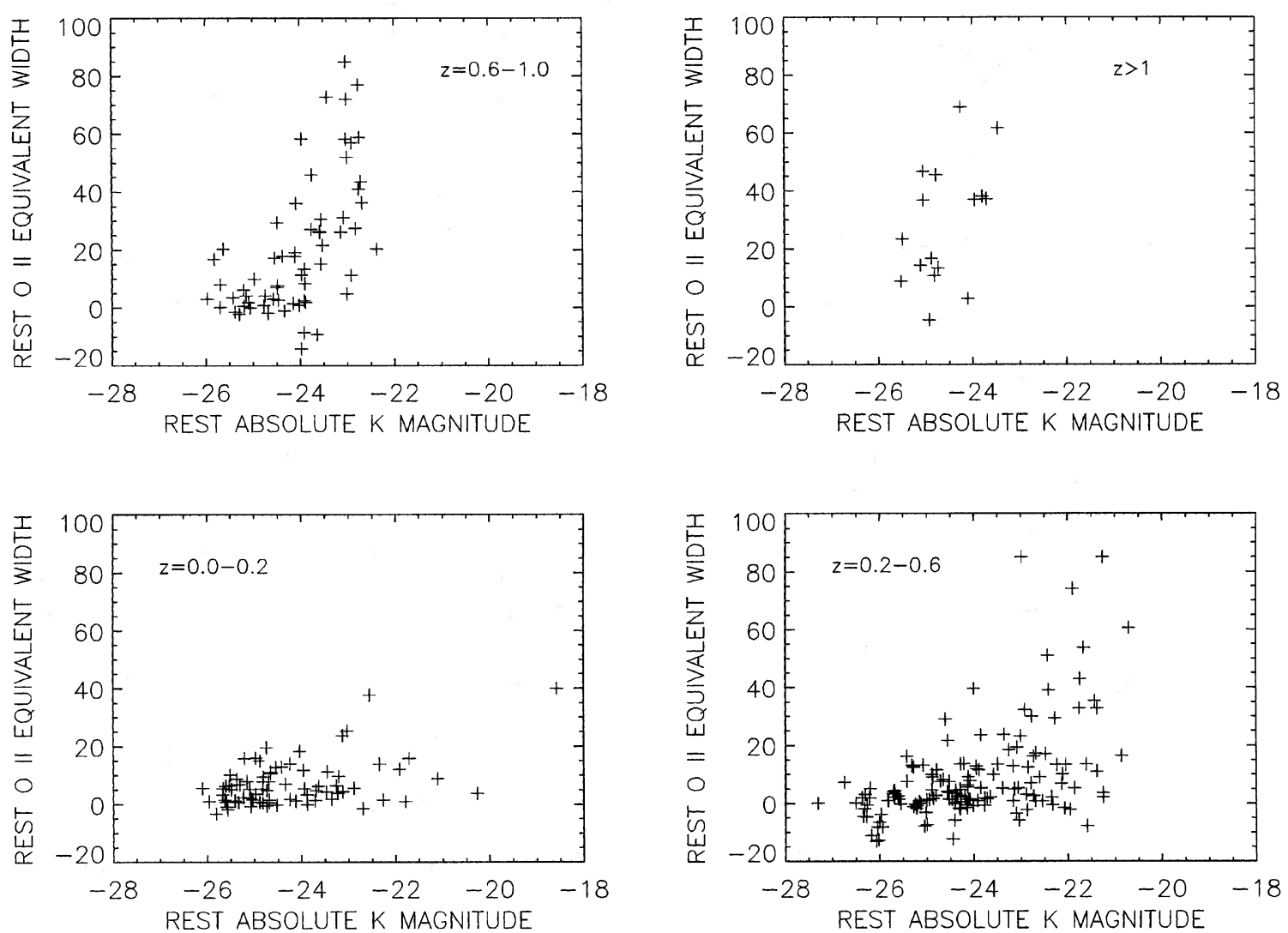

FIG. 10. Rest-frame [O II] equivalent width versus absolute rest $K$ magnitude and redshift for the $K<20$ sample. In the lowest redshift interval (lower left panel) very few galaxies have strong [O II $]$ lines or are undergoing rapid star formation $(\mathrm{EW}([\mathrm{O} \mathrm{II}]) \gtrsim 25 \AA)$. At higher redshifts, progressively more massive galaxies are undergoing rapid formation, until at $z>1$ the locus of rapidly forming galaxies reaches $M_{K *} \sim-25$.

magnitude relation computed for a no-luminosity-evolution model with the $B$ luminosity function of Loveday et al. (1992), the galaxy type mix of King \& Ellis (1985) and the $K$ corrections of Fig. 1 of Cowie et al. (1991). This prediction agrees well with that of Glazebrook et al. (1995a), but is significantly higher than that of Colless et al. (1993) apparently because the analytic approximation to the $K$ corrections used in the latter paper is poor at higher redshifts. This noevolution prediction provides a reasonable fit to the data. The dashed line shows the Carlberg (1992) merger model, which slightly under-predicts the median redshifts at fainter $B$ magnitudes.

Previously published $B=24$ samples have either been rather small (Cowie et al. 1991) or relatively incomplete. The large $B=24$ sample of Glazebrook et al. (1995a) contained 157 objects of which 84 were galaxies with secure redshifts, 8 were stars, and 2 were quasars - the remaining 63 objects were unidentified, though a smaller subsample was much more complete. The present sample contains a very comparable number of $B<24$ objects but is much more complete than Glazebrook et al., with 149 of the 156 objects identified. The redshift distribution of identified objects in the two samples in the $B=22.5-24$ range is compared in Fig. 13, where the present sample is shown as the solid histogram and the Glazebrook et al. (1995a) sample (hereafter
GECBAT) is shown as the dashed histogram. At $z<0.7$ the shapes of the two histograms agree well but the present data shows an extended tail beyond this redshift which is not seen in the GECBAT data.

Most of the objects in the high redshift tail are blue $(B-I)$ galaxies with extremely strong [O II] emission. (A number of such objects are shown in Figs. 2(a) and 2(b) and further examples may be found in Fig. 1 of Cowie et al. (1995a), in Lilly et al. (1995a), and in LeFèvre et al. (1994).) This is illustrated in Fig. 14, where we show the histogram of the redshift distribution cut by the color criterion $(B-I)=1.6$. The blue population divides neatly into a low redshift $z \sim 0.25$ population and a high redshift $z>0.8$ population, while the red population lies at intermediate redshifts. This allows us to understand the difference between GECBAT and the present data. Most of the unidentified objects in GECBAT correspond to the blue population (cf. GECBAT. Fig. 3) and, given that $z \sim 0.2$ blue galaxies have strong emission lines in the observed optical and are easily identified, the bulk of these missing objects will be drawn from the high- $z$ tail. GECBAT's upper wavelength cutoff was around $8000 \AA$ so that for objects with $z>1.1$, the [O II] line is outside their spectral window and such objects would not have been identified. GECBAT argued that such objects would have been present in the Colless et al. (1993) sample 

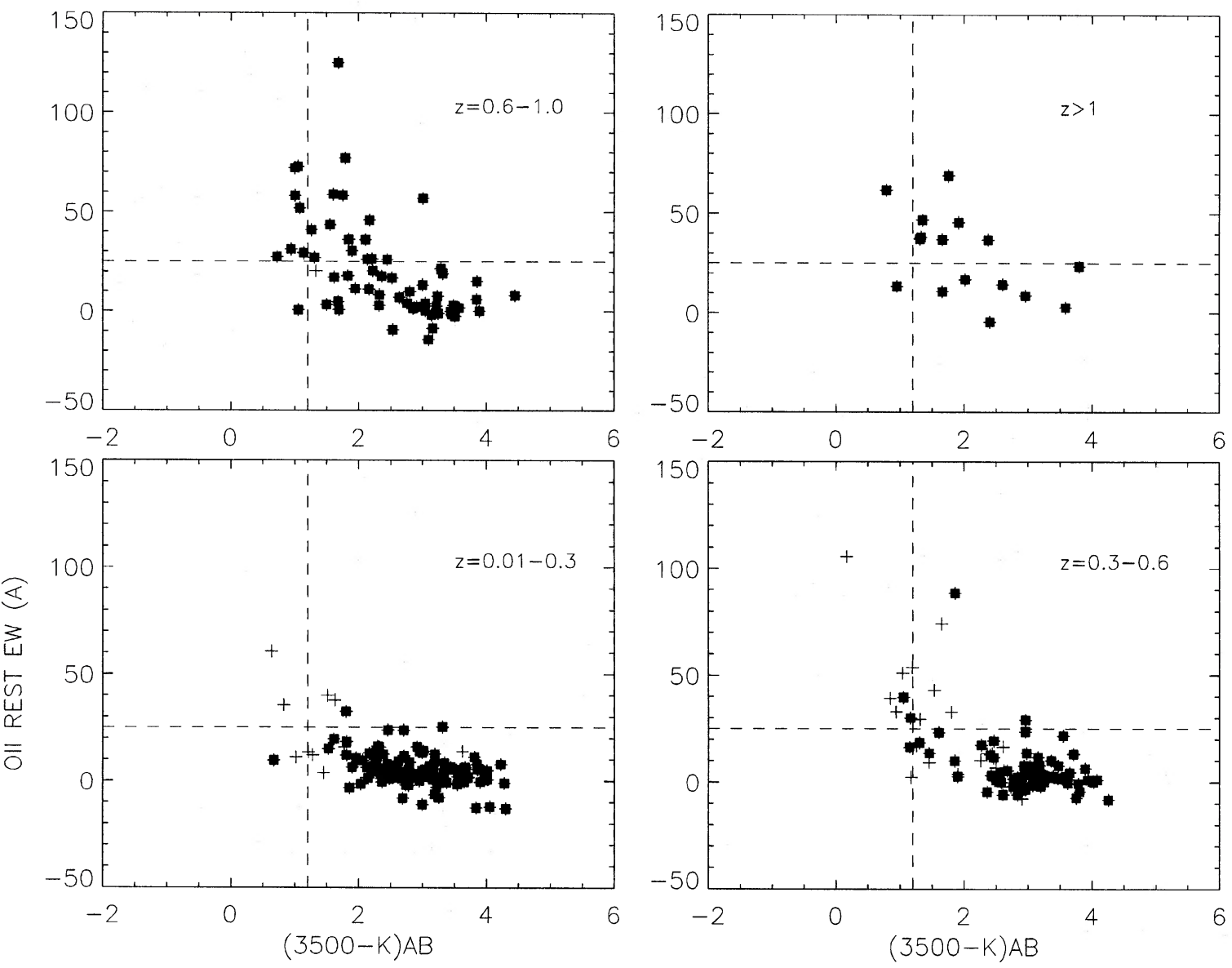

FIG. 11. Comparison of the rest-frame equivalent width of [O $\mathrm{II}] 3727$ in angstroms with the rest-frame $(3500-K)$ color in $A B$ magnitudes for various redshift slices. Pluses are galaxies with $M_{K}>-22$, filled squares superposed on pluses mark more luminous galaxies. Galaxies with strong star formation should have $\mathrm{EW}([\mathrm{O}$ II $]) \geq 25 \AA$ and $(3500-K)_{A B} \leq 1.3$ (dashed lines).

of blue-selected galaxies, where 7 of 13 galaxies with $(B-$ $I)<1.6$ were identified and found to be at modest redshift (all of the $(B-I)<1$ objects being so identified). The Colless et al. galaxies are mostly drawn from brighter magnitudes where the preponderance of blue objects may be more

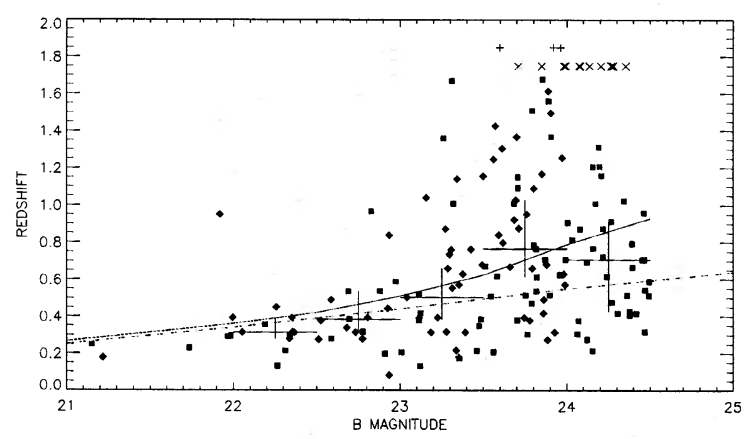

FIG. 12. Redshift vs apparent $B$ magnitude. Diamonds=SSA 13 field to $B=24$, squares $=$ SSA 22 field to $B=24.5$. The crosses and pluses show unobserved or unidentified objects at a nominal redshift. The large crosses show the median redshifts with $\pm 1 \sigma$ error bars in half-magnitude intervals compared to a no-luminosity evolution model described in the text (solid line) and a merger model (Carlberg 1992) shown as a dashed line.

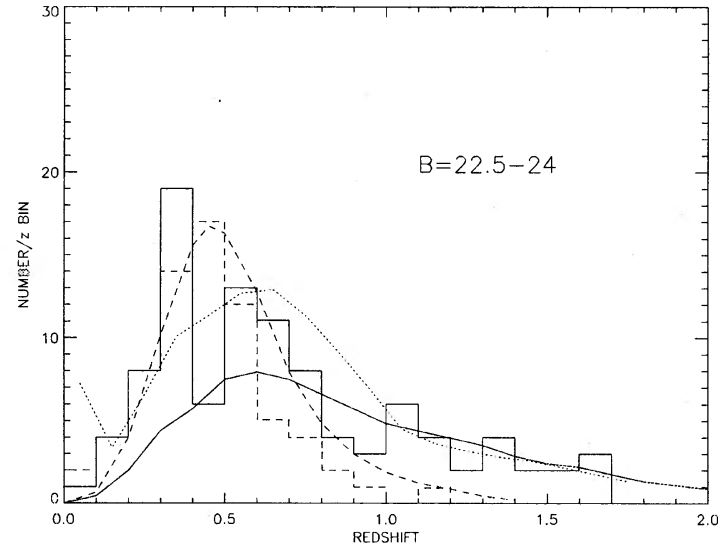

FIG. 13. Histogram of the redshift distribution of $B=22.5$ to $B=24$ galaxies in the present sample (solid histogram) compared with that of Glazebrook et al. (1995a) (dashed histogram). Also shown are the predictions of a luminosity evolution model (solid line), a merger model (Broadhurst et al. 1992; dashed line) and a steep dwarf luminosity function model (Gronwall \& Koo 1995; dotted line). The latter model predicts a substantial number of faint blue objects at $z<0.1$, corresponding to a large population of dwarf galaxies, which are not seen in the present sample. 


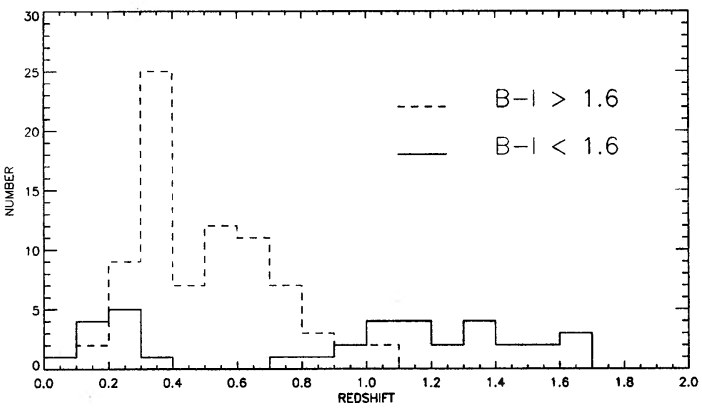

FIG. 14. $B=22.5$ to $B=24$ redshift histogram divided by $(B-I)$ color. (Solid line: $(B-I)<1.6$, dashed line: $(B-I)>1.6$.) Most of the objects in the high-redshift tail $(z \geqq 0.8)$ are blue $[(B-I)<1.6]$ and show strong [O II] emission. The $(B-I)$ color criterion is shown to divide the blue population into low-redshift $(z \sim 0.25)$ and high-redshift $(z \geqq 0.8)$ components, with the red population lying at intermediate redshifts.

weighted to low redshifts (cf. Sec. 4.3), but this sample is also sufficiently small so that the redshift distribution of its blue objects is not inconsistent with the result from the present sample, which is that 11 of 36 objects with $(B-I)$ $<1.6$ are low- $z$ objects and 25 of the 36 are at high $z$. We therefore conclude that the present data are indeed consistent with previous results when systematic effects in the incompleteness are allowed for.

\subsection{Local Faint-end Blue Luminosity Function}

Shanks (1990) suggested that there might be a steep rise in the local faint-end blue luminosity function, and Koo and his collaborators have attempted to provide a self-consistent fit to the number counts, colors and $B$-band redshift information by including such a steeply rising population of blue dwarf galaxies at the faintest $B$ magnitudes $\left(M_{B} \leqslant-16.75\right)$. Glazebrook et al. (1995a) rejected earlier versions of this model based on their $B=24$ sample. Here we shall consider the most recent version of this model described in Gronwall $\&$ Koo (1995). Because of the large number of dwarfs, these models predict that in $B=24$ samples we should see a substantial number of galaxies at $z \leqslant 0.1$. Figure 13 shows this for the Gronwall \& Koo (1995) model, where it is compared with the Glazebrook et al. sample and our own. We find only one $B \leqslant 24$ galaxy with $z<0.1$ while Glazebrook et al. find 2 -considerably lower than this model predicts.

To quantitatively test the model, we predicted the expected number of $B<24, z<0.1$ objects in the two areas, using the luminosity function of objects tabulated in Gronwall \& Koo's (1995) paper. We obtained a predicted value of 8 in the current sample and 12 in the Glazebrook et al. sample. Either sample rejects the Gronwall-Koo prediction at a high confidence level while in the combined sample the probability of the Gronwall-Koo luminosity function being acceptable is only $3 \times 10^{-6}$ ( 3 objects observed versus 20 predicted).

Next we directly constructed the low-end $B$-band luminosity function $\left(M_{B}<-20\right)$ using the $1 / V$ method with the combined $z<0.2$ sample. Because almost all of these objects are extremely blue, there is only a very small $K$ correction over this redshift interval. The results are shown in Fig. 15,

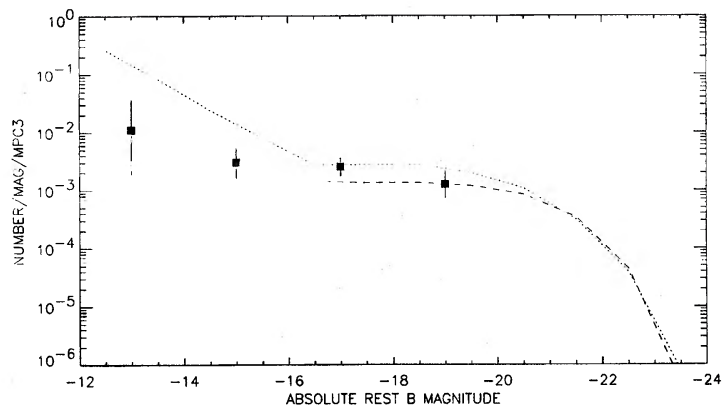

Fig. 15. Local faint-end $B$ luminosity function (filled squares with $1 \sigma$ error bars) computed from the present data and Glazebrook et al. (1995a). The dashed line shows the luminosity function determined by Loveday et al. (1992) and the dotted line the function assumed by Gronwall \& Koo (1995). The data are consistent with a smooth extension of the Loveday et al. function to brighter magnitudes or with a shallow rise (Eales 1993), but are not consistent with either the Gronwall-Koo function or the steep rise given by Shanks (1990) at the bright end.

where they are compared with the Loveday et al. (1992) luminosity function (dashed line) and with the Gronwall-Koo function (dotted line). The data are marginally consistent with a smooth extension of the Loveday et al. function but are better fit by a shallow rise (Eales 1993). However, they are not consistent with the Gronwall-Koo function.

\subsection{Colors and Redshifts versus B Magnitude}

In Fig. 16, we show the distribution of redshift versus $(B-I)$ color in various $B$ magnitude slices. Objects with red $(B-I)$ colors $(\gtrsim 1.6)$ lie at intermediate redshifts and show a fairly smooth increase in median redshift with increasing magnitude. However, the redshift distribution of $(B-I)<1.6$ objects is more complex. At $B<23$ such objects lie primarily at $z \sim 0.25$ but at $B \sim 24$ most lie at $z \geqq 0.8$ with a fraction at $z \sim 0.25$. This extended distribution can also be clearly seen in Fig. 14. Most of the unidentified objects also lie in this color range and may correspond to objects where the [O II] 3727 line was moved out of the observed spectral range $(z \gtrless 1.6)$. In Fig. 17 we show the position of the galaxies as a function of redshift in the $(B-I)$ versus $(I-K)$ color-color plane, which also illustrates that most of the unidentified objects (open diamonds) lie at the same colors as the $z>1$ galaxies (filled diamonds).

\section{THE $I$ SAMPLE

$$
5.1 I-z
$$

The $I-z$ relation is given in Fig. 18, with filled squares showing the SSA 22 field to $I=23$ and diamonds SSA 13 to $I=22.5$. The small number of objects which were not observed or not identified are shown as plus signs for SSA 22 and crosses for SSA 13. The large upright crosses show the median redshifts with $1 \sigma$ error bars computed using the median sign method and allowing unidentified or unobserved objects to be at either high or low redshift.

The CFRS survey has produced a very large, spatially sparse sample of galaxies to $I=22$ whereas the present sample contains all galaxies in our areas to this magnitude. The areal densities of objects versus redshift in the two 


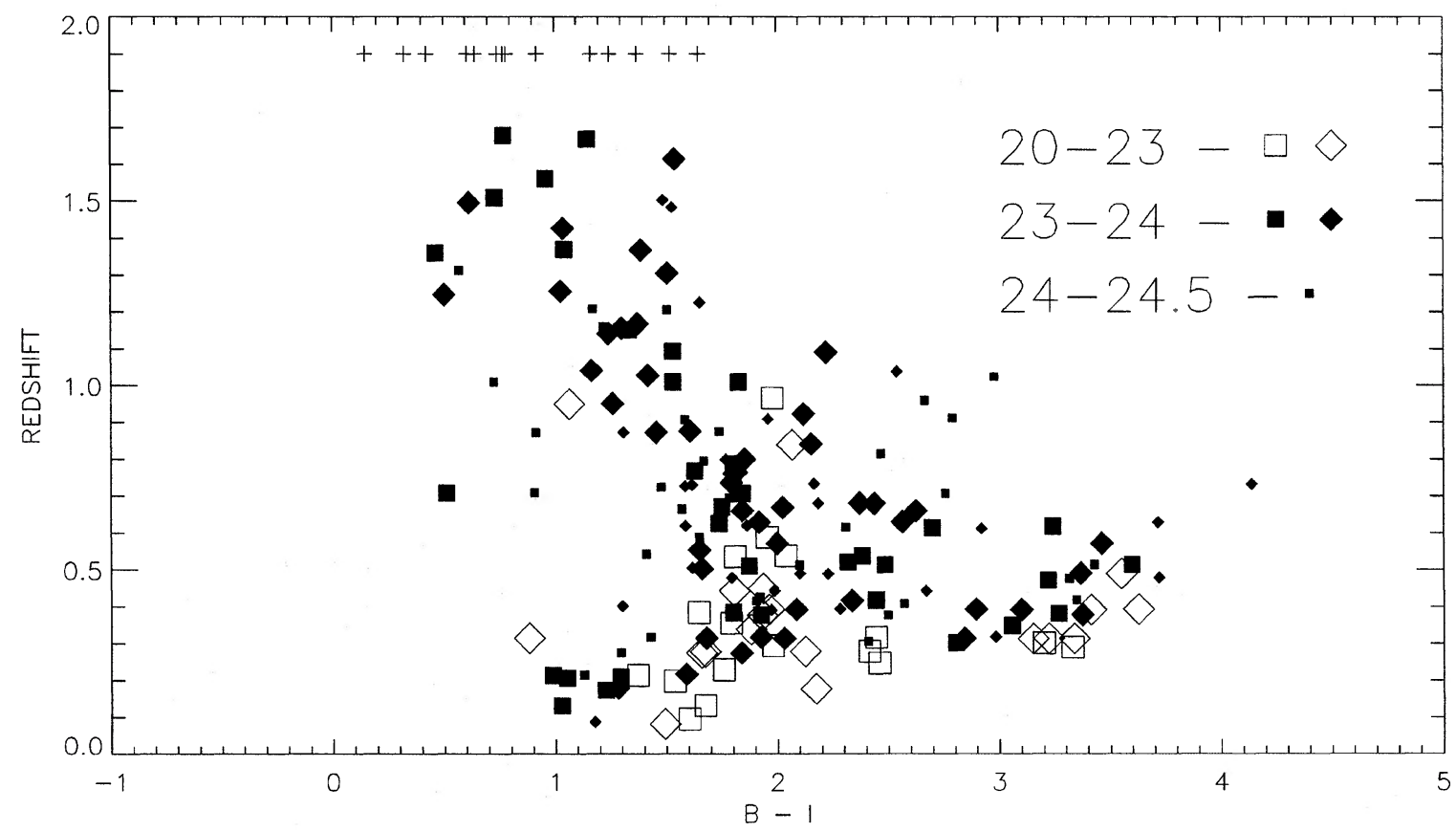

Fig. 16. Redshift vs $(B-I)$ color and $B$ magnitude. Squares are from the SSA 22 field and diamonds from the SSA 13 field. Pluses show unidentified objects at a nominal redshift. Objects with red $(B-I)$ colors $(\geqq 1.6)$ lie at intermediate redshifts. The bimodal distribution of blue $[(B-I)<1.6]$ objects into low- $(z \sim 0.25)$ and high-redshift $(z \geq 0.8)$ populations shown in Fig. 14 may be seen in more detail here, and is seen to onset at $B \sim 23$. Most of the unidentified objects (pluses) also lie within this range of blue $(B-I)$ colors, and many of these may correspond to objects where the [O II] 3727 line moves out of the observed spectral range $(z \gtrless 1.6)$.

samples for $I=17-22$ are compared in the upper panel of Fig. 19 and agree strikingly well in shape, and more approximately in normalization, with the present sample-being about $20 \%$ below the CFRS value. Lilly et al. (1995b) have used the CFRS sample to predict the redshift distribution at fainter magnitudes and we compare this (lower panel: dotted line) with the observed distribution at $I=22-23$. This CFRS extrapolation has a similar shape to the observed $I=22-23$

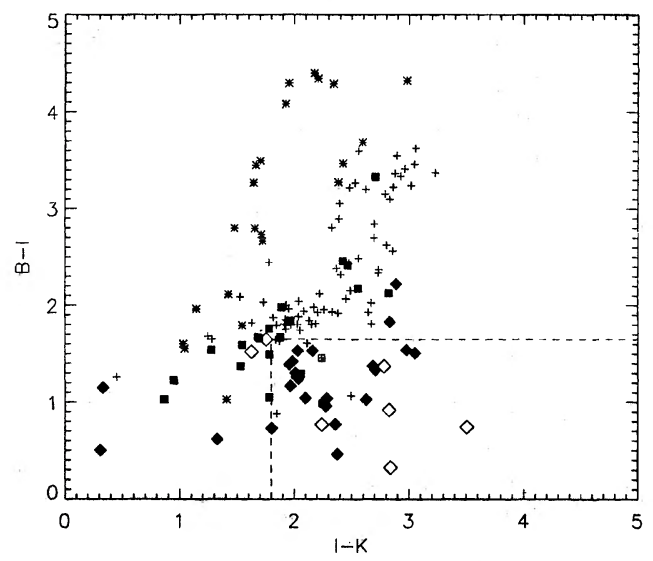

FIG. 17. Color-color diagram of the $B$-selected sample showing the positions of stars (asterisks), $z<0.3$ galaxies (filled squares), $0.3<z<1$ galaxies (pluses), $z>1$ galaxies (filled diamonds) and unidentified objects (open diamonds). Most of the unidentified objects have blue $(B-I)$ colors and redder $(I-K)$ colors and lie in the portion of the color-color plane (dashed lines) where most of the $z>1$ galaxies (filled diamonds) also lie. sample, but is slightly overweighted to high redshift objects.

The CFRS includes 28 objects with $I<22$ that lie in the present SSA 22 field region. All of these objects are contained in the present catalog and the $I$ magnitudes are in good agreement, with $\left\langle I-I_{A B}^{\mathrm{CFRS}}\right\rangle=0.5 \pm 0.25$, as expected. The redshifts given in the two surveys agree in all cases where a CFRS redshift was obtained. Even in the one case where an object was excluded from the CFRS analysis with

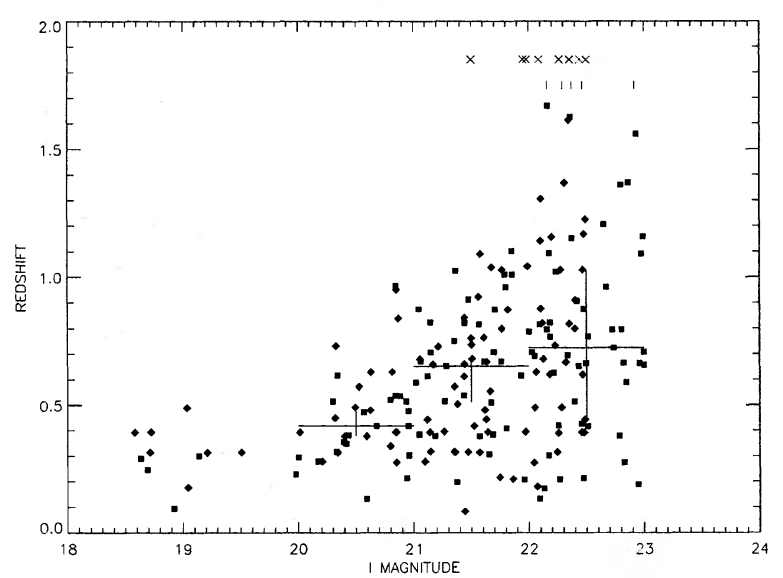

FIG. 18. Redshift vs $I$ magnitude with filled squares showing data for the SSA 22 field $(I<23)$ and filled-diamonds the SSA 13 field $(I<22.5)$. Pluses and crosses are unidentified or unobserved objects placed at a nominal redshift. The large upright crosses show median redshifts with $1 \sigma$ errors computed using the median sign method. 

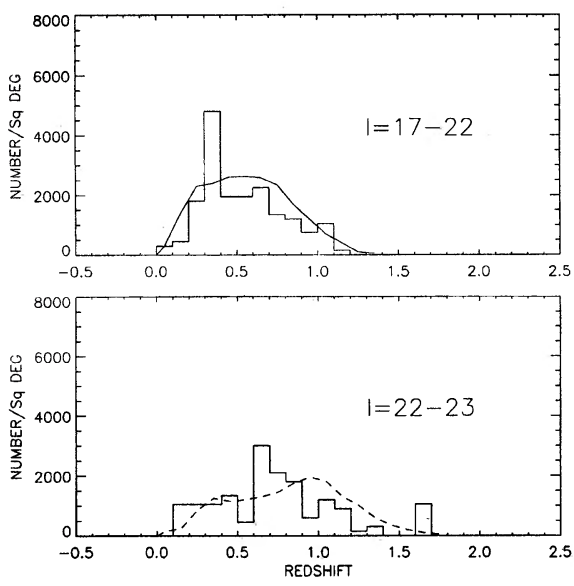

Fig. 19. (Upper panel) Comparison of the surface density of objects vs redshift (histogram) in the present sample with $I=17-22$ with that in the CFRS (solid line). (Lower panel). Comparison of the present data with $I=22-23$ (histogram) vs the no-evolution prediction from CFRS (Lilly et al. 1995b).

a low confidence ( 22.1210 with a value of 1$)$ the CFRS redshift agrees with the present value.

\subsection{The High-z Rest-frame B-band Luminosity Function}

Following Lilly et al. (1995b), we have used the I-band sample to create the rest-frame $B$-band luminosity function directly at $z \sim 0.8$, where the observed $I$ corresponds to restframe $B$. At this redshift the rest-frame absolute $B$ magnitude $M_{B}$ is given by

$$
M_{B}=I-d m+1.4,
$$

where $d m$ is the distance modulus and the final term is the $K$ correction allowing for the relative wavelengths and zero points. For neighboring redshifts, there is a small typedependent $K$ correction to equation (6) which we have assigned using an approximate type derived from the color and redshift, following the procedures outlined in Sec. 3.3. However, the results are essentially unaffected by this small correction.

The rest-frame $B$-band Schechter function, computed from the $I$ sample using the $1 / V$ method in the redshift interval $z=0.6-1.0$, is shown in the upper panel of Fig. 20 as the large open boxes. Downward pointing arrows denote a $1 \sigma$ upper limit while $\pm 1 \sigma$ error bars are superimposed on the symbols, assuming Poisson errors based on the number of objects in each bin. The solid line shows a fit to the points with the assumption that the luminosity function has the same shape as the local value $\left(M_{B *}=-21.0+5 \log _{10} h_{50}\right.$, $\alpha=-0.97)$ derived by Loveday et al. (1992). This provides an acceptable fit to the data with $\phi_{*}=4.1 \times 10^{-3} h_{50}^{3} \mathrm{Mpc}^{-3}$ compared to the local value of $1.8 \times 10^{-3} h_{50}^{3} \mathrm{Mpc}^{-3}$ of Loveday et al. which is shown as the dashed line. The rise in normalization is similar to that derived by Eales (1993).

For comparison we have also shown the corresponding luminosity function derived by Lilly et al. (1995b) for $z=0.5$ -0.7 (open triangles) and $z=0.7-1.0$ (open diamonds). These fall somewhat above the present data, reflecting the slightly higher normalization in the histogram of Fig. 19. We have also shown (filled squares) the local faint-end $B$-band luminosity function derived in Sec. 4.2.

The lower panel also shows the relative rest-frame $K$-band luminosity functions both locally and at $z=0.6-1$. The infrared normalization rises by a considerably smaller amount (1.25). In combination the two results show that the rest-frame light is considerably bluer at $z=1$ (by about 0.6 mag in the rest frame $(B-K))$, which is consistent with the discussion of the colors and line strengths of the $K$ sample in Sec. 3.3. However, Fig. 20 makes the important point that at these redshifts the evolution appears to be primarily in the $L<L_{*}$ galaxies and not to be affecting the colors or luminosities of the near- $L_{*}$ galaxies; this reflects the differential luminosity evolution we shall discuss in the next section.

\section{INTERPRETATION}

$$
6.1 M_{K}-t_{d}-z
$$

Each galaxy in the sample can be roughly characterized by three quantities, $z$ the redshift, $M_{K}$, which for older galaxies is a rough measure of the total mass in stars, and either an emission-line equivalent width $[E W([O \mathrm{II}])$ or $E W(H \alpha)]$ or a rest-frame ultraviolet color $\left[\phi_{\nu}(3500) / \phi_{\nu}(21000)\right.$ $\equiv U K(A B)]$, which roughly measures the rate of star formation relative to the existing stellar mass. The rest-frame ultraviolet-infrared color is a more direct measure of the stellar properties than the emission line strengths (Kennicutt 1992 ), and here we use $U K(A B)$ rather than equivalent widths. For galaxies with little internal opacity $U K(A B) \equiv(3500-21000)_{A B} \equiv-2.5 \log _{10} \phi_{\nu}(3500) / \phi_{\nu}(21000)$ is a monotonic function of the stellar mass doubling time, as is illustrated in Fig. 21, which is computed from the models of Bruzual \& Charlot (1993) for a Salpeter IMF. Galaxies with $U K(A B) \leqslant 1.3$ have doubling times less than $10^{10}$ years and may be thought of as "in formation," where formation here means only that the bulk of stars would be made if the process persisted for a fraction of the local Hubble time, and does not necessarily mean that there has not been earlier star formation. Uncertainties in the $U K(A B)$ color of $0.3 \mathrm{mag}$, which represents the maximum deviation between the two interpolation methods discussed in Sec. 3.3, would introduce uncertainties of about a factor of 2 in $t_{d}$ for $t_{d} \sim 10^{10} \mathrm{yrs}$. However, a more important source of uncertainty is internal extinction. Internal extinction implies that the doubling time is overestimated using the observed $U K(A B)$. For a typical internal extinction of $\mathrm{E}(B-V)=0.2$ to $\mathrm{H}$ II regions in blue irregulars (Gallagher et al. 1989), the intrinsic $U K(A B)$ would be roughly a magnitude bluer than the observed value, with a corresponding reduction in $t_{d}$.

The position of each galaxy in the three-dimensional $M_{K}-t_{d}-z$ space is shown in Fig. 22, which synthesizes the first main conclusion of the present paper (see Sec. 3.3 for a more phenomenological version). We can summarize the figure as follows: At $z<0.2$ there are no forming galaxies above $M_{K}=-20+5 \log _{10} h_{50}\left(0.01 L_{*}\right)$. At $z=0.2-0.4$, we see forming galaxies at $M_{K}=-20$ to $-22.5+5 \log _{10} h_{50}$ $\left(0.01-0.1 L_{*}\right)$. At $z=0.4-0.8$ forming galaxies are seen 

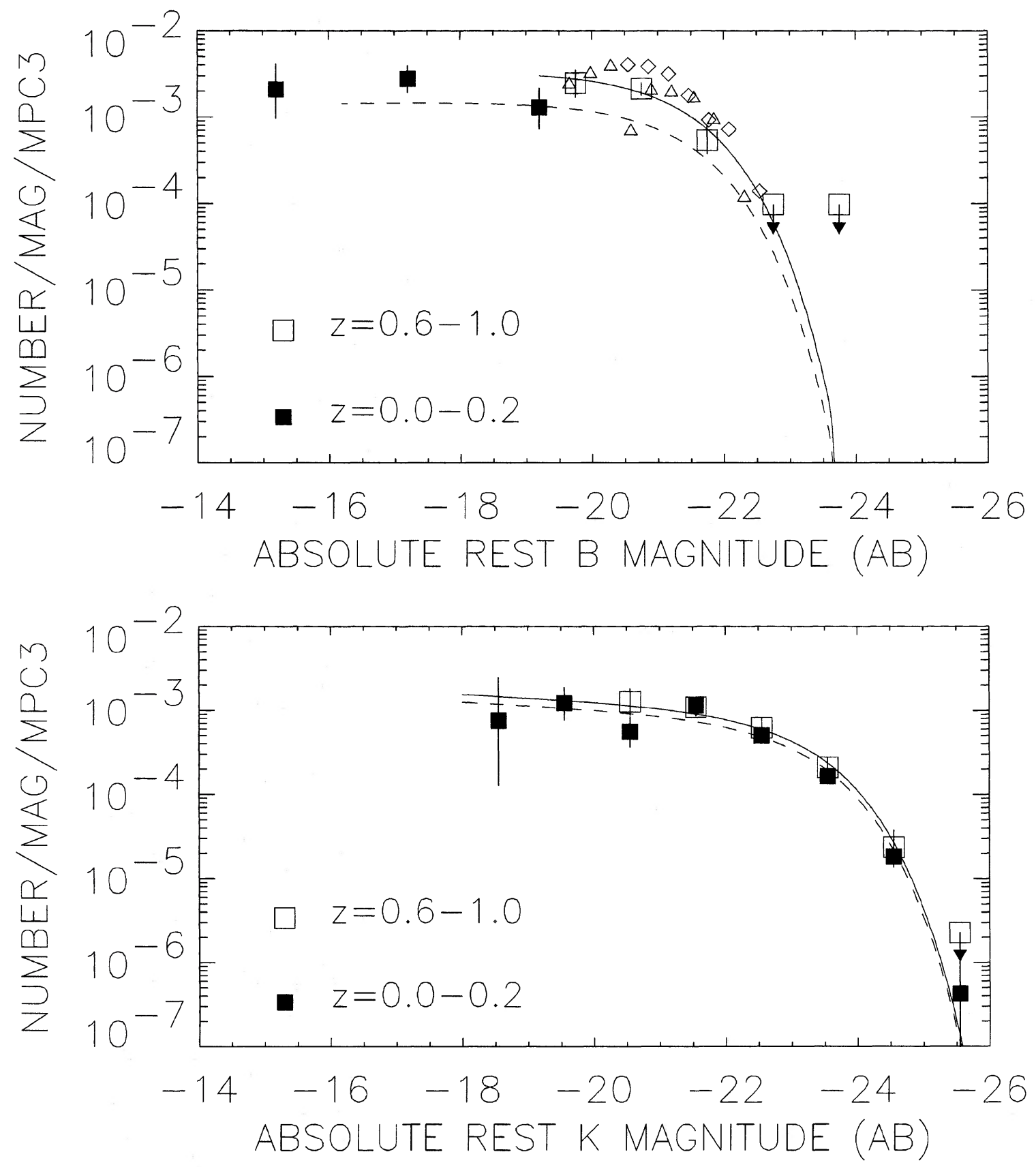

FIG. 20. Comparison of the rest $K$-(lower) and rest $B$-band luminosity functions at $z=0-0.2$ (filled squares) and $z=0.6-1$ (open squares). In the upper panel we show similar results for the CFRS at $z=0.5-0.7$ (open triangles) and $z=0.7-1$ (open diamonds). The dashed line in the upper panel shows the Loveday et al. (1992) Schechter function and the solid line a Schechter function with the same shape but normalized to the high redshift data. The lower panel has similar fits for $M_{K *}=-25.0, \alpha=-1.25$, and shows a considerably smaller rise in the infrared normalization (factor of 1.25 ) between the low- and highredshift data (dashed and solid curves) than is seen for the $B$ data in the upper panel. The data in combination show that the rest-frame light is considerably bluer at $z \sim 1$ (see, e.g., Figs. 14 and 16), and that at these redshifts the evolution is primarily in sub- $L_{*}$ galaxies, while the colors and luminosities of near- $L_{*}$ galaxies are not substantially affected.

which are as bright as $M_{K}=-23.5+5 \log _{10} h_{50}$, and at $z=0.8$ to $z=1.6$ as bright as $M_{K} \sim-24+5 \log _{10} h_{50}$.

The time interval between the redshift slices is roughly $10 \%$ to $15 \%$ of the current age of the universe, depending on the assumed geometry, and it is clear that there is substantial migration in the $t_{d}-M_{K}$ space between each redshift inter- val. As is illustrated in Fig. 22 for the Bruzual-Charlot models for various exponential star-formation models ( $\tau=3 \mathrm{Gyr}$ dotted, $\tau=7$ Gyr-solid, and constant SFR-dashed), it is clear that this migration requires that the SFR be declining faster than a 3 Gyr exponential to move the fast-forming galaxies away from their initial locus. This result may be weakened 


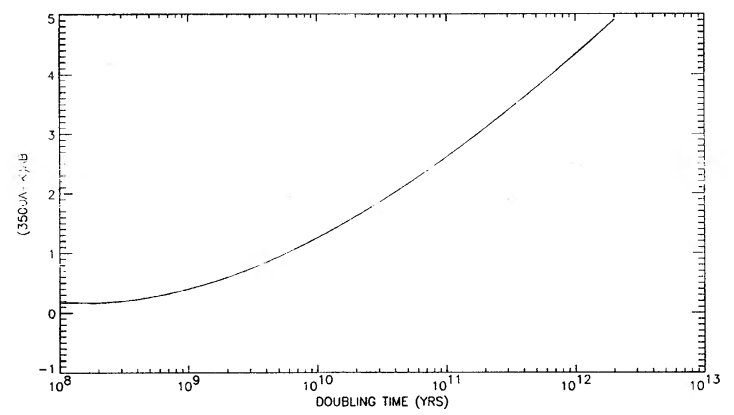

FIG. 21. Relation between rest-frame $(3500-K)_{A B}$ and the stellar mass doubling time, computed from the models of Bruzual \& Charlot (1993). Galaxies with $(3500-K)_{A B} \lesssim 1.3$ have doubling times less than $10^{10}$ years. If there is significant internal extinction the plotted relation will overestimate the stellar mass doubling time for galaxies.

somewhat if the evolution is accompanied by an increase in internal extinction.

Figure 22 leaves open the question of the final location of the galaxies which could lie at magnitudes only slightly fainter than their initial $M_{K}(\tau=3$ Gyr exponential SFR) in which case we are seeing the formation of the massive end of the Schechter function, or at several magnitudes fainter $\left(10^{8}\right.$ yr burst) in galaxies that were only transiently luminous. This question is best addressed in terms of the distribution and evolution of the $K$ light density and the UV light density production, to which we now turn.

\section{$6.2 \mathrm{~K}$-band Light Density Evolution}

The rest-frame $K$-band light density provides our best available tracer of the baryonic mass which has formed into stars. The present day $K$-band light density of $2.5 \times 10^{8} L_{\odot}$ to $M_{K}=-20+5 \log _{10} h_{50}\left(L=0.01 L_{*}\right)$, as measured in Fig. 6(a), translates to a stellar baryonic mass density of $2 \times 10^{8} h_{50} M_{\odot}$ $\mathrm{Mpc}^{-3}\left(\Omega=3 \times 10^{-3} h_{50}^{-1}\right)$ for a mass-to- $K$-light ratio of 0.8 in solar units. As is obvious from the shape of the $K$-band Schechter function, more than $85 \%$ of the light lies in luminous galaxies $\left(M_{K}=(-22 \rightarrow-26)+5 \log _{10} h_{50}\right)$.

In Fig. 23 we have constructed the $K$-band cumulative luminosity density for the $K=20$ selected sample for $z=0.0$ -0.3 and $z=0.3-1$, as was done in Fig. 6(a), but have now split it into $t_{d}<10^{10} \mathrm{yr}$ and $t_{d}>10^{10} \mathrm{yr}$ samples $(U K(A B)$ $<1.3$ and $U K(A B)>1.3$ ). In each case the old (red)

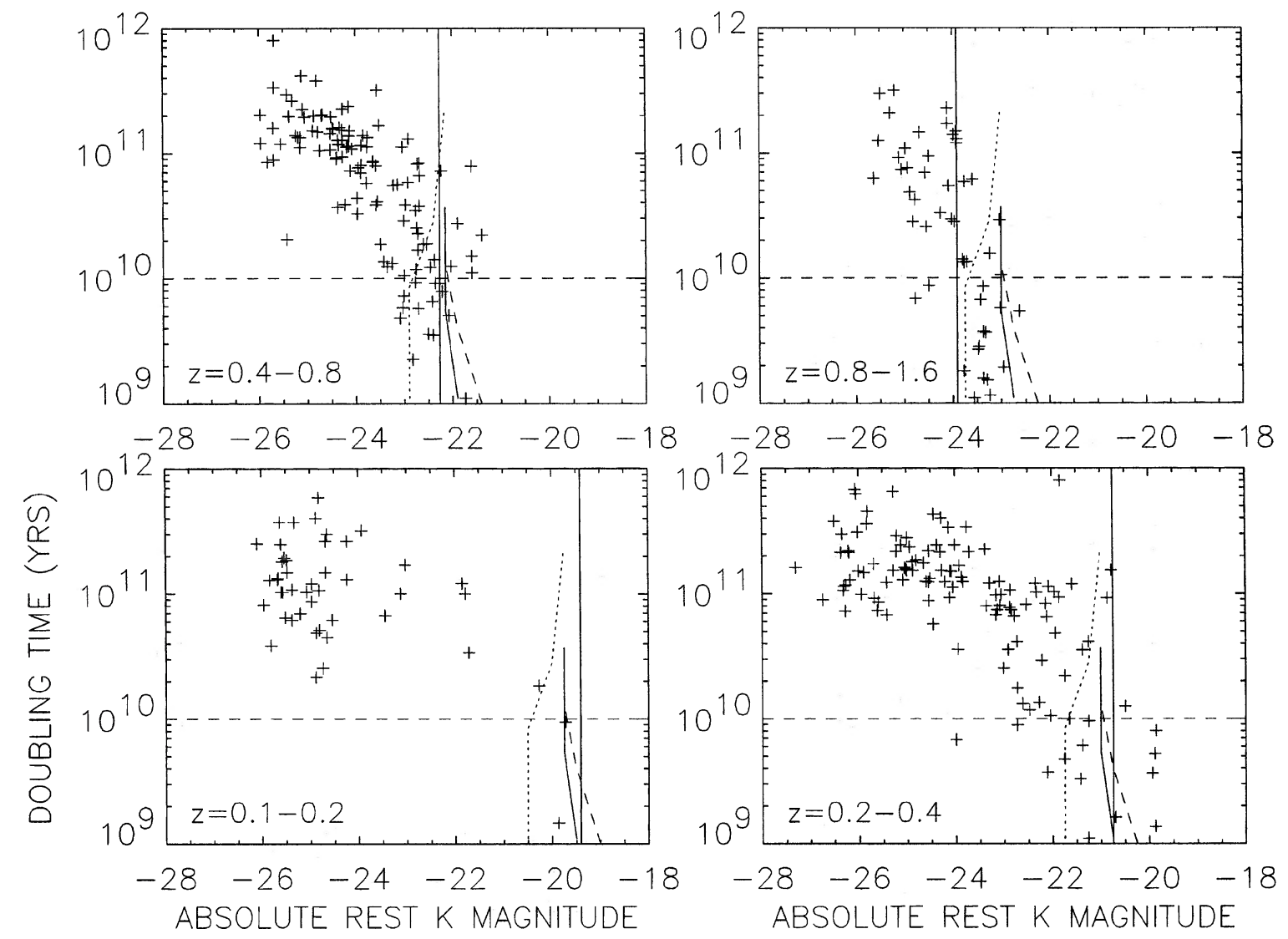

Fig. 22. Doubling time versus rest absolute $K$ magnitude as a function of redshift for all identified galaxies in the sample with $K \leqslant 20$. The solid vertical line shows the absolute magnitude detection limit at the center of the redshift interval, and the dashed horizontal line marks a fiducial doubling time of $10^{10}$ years, below which value galaxies may be thought of as "in formation." The various tracks show the evolution of galaxies with exponentially declining starformation rates over a period of $13 \mathrm{Gyr}$ computed from the models of Bruzual \& Charlot (1993). Dotted: $\tau=3 \mathrm{Gyr}$, solid: $\tau=7 \mathrm{Gyr}$, dashed: constant star formation rate. The migration of rapidly star-forming galaxies from their original locus across this plane with changing redshift interval suggests that in this simple model (ignoring internal extinction) the star formation rate is declining more rapidly than it would for a 3 Gyr burst model. 


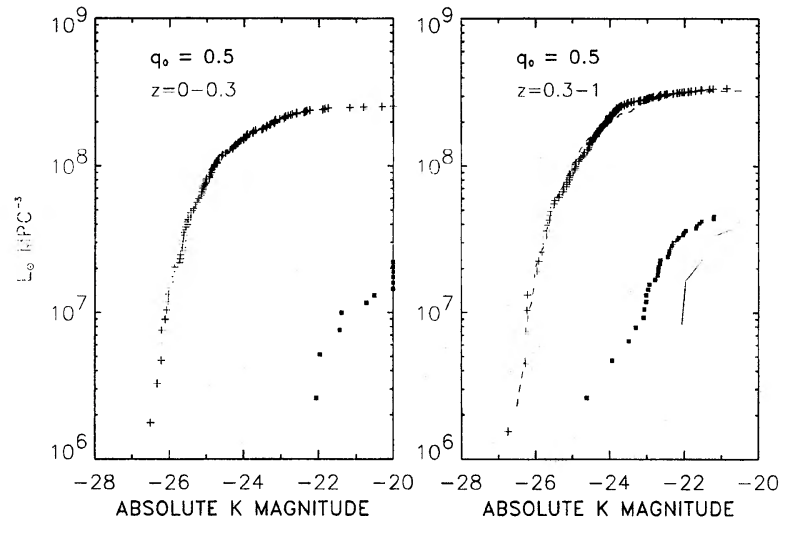

FIG. 23. $K$-band light density $(K \leqslant 20$ sample) of galaxies in the redshift intervals $z=0-0.3$ (left panel) and $z=0.3-1$ (right panel). Both are computed for $q_{0}=0.5$. The cumulative luminosity function of Fig. 6 a has been split into samples of red galaxies $(U K(A B)>1.3$, pluses) with doubling times $t_{d}>10^{10}$ years and blue galaxies $(U K(A B)<1.3$, filled squares) with doubling times $t_{d}<10^{10}$ years. The fraction of light in forming objects is considerably higher for the higher redshift interval (right panel), and extends to brighter galaxy magnitudes. The solid curve shows the shape of the cumulative luminosity density from the forming galaxies of the lower redshift interval (left panel) renormalized to the higher redshift curve, and may be compared with the curve for forming galaxies (filled squares) in the right panel.

sample is shown by crosses and the young (blue) sample by boxes. In the lower redshift bin $5 \%$ of the light to $M_{K}=-20+5 \log _{10} h_{50}$ comes from the young objects and these are all faint $\left(M_{K}\right.$ fainter than $\left.-22+5 \log _{10} h_{50}\right)$. However, in the higher redshift bin the fraction of light in forming objects is considerably higher $\left(10 \%\right.$ to $\left.M_{K}=-21\right)$ and also extends to considerably brighter magnitudes. For comparison we have shown as a solid line the shape of the cumulative luminosity density in the lower redshift interval renormalized to the higher redshift curve, which shows clearly that the extension to brighter magnitudes is not simply an effect of the higher normalization but represents a brightening of the luminosity of forming galaxies at higher redshift.

This relatively rapid evolution in blue-selected versus redselected samples has also been emphasized by Lilly et al. (1995b) based on the CFRS I-band sample and the same effect has been found by Ellis et al. (1996) using line diagnostics. Ellis $e t$ al. find that the luminosity function of strong [O II] line emitters evolves rapidly but that of weak [O II] emitters does not. It is probable that the rapid increase in the fraction of galaxies with unusual morphologies at increasing $z$ (Glazebrook et al. 1995b; Cowie et al. 1995b; Driver et al. 1995) also reflects this underlying evolution.

The luminosity function of the old (red) galaxies shows very little evolution of its shape over this redshift interval, and with the exclusion of the young objects which are much more rapidly evolving, it is found that the red-selected sample evolves less in light density than the total sample. From Fig. 23, $\lambda_{K}$ to $M_{K}=-21$ has changed from $3.3 \times 10^{8}$ $L_{\odot} \mathrm{Mpc}^{-3}$ for $z=0.3$ to 1 , to $2.5 \times 10^{8} L_{\odot} \mathrm{Mpc}^{-3}$ for $z=0$ -0.3 , for $q_{0}=0.5$, while for $q_{0}=0.02$ this becomes $2.6 \times 10^{8}$

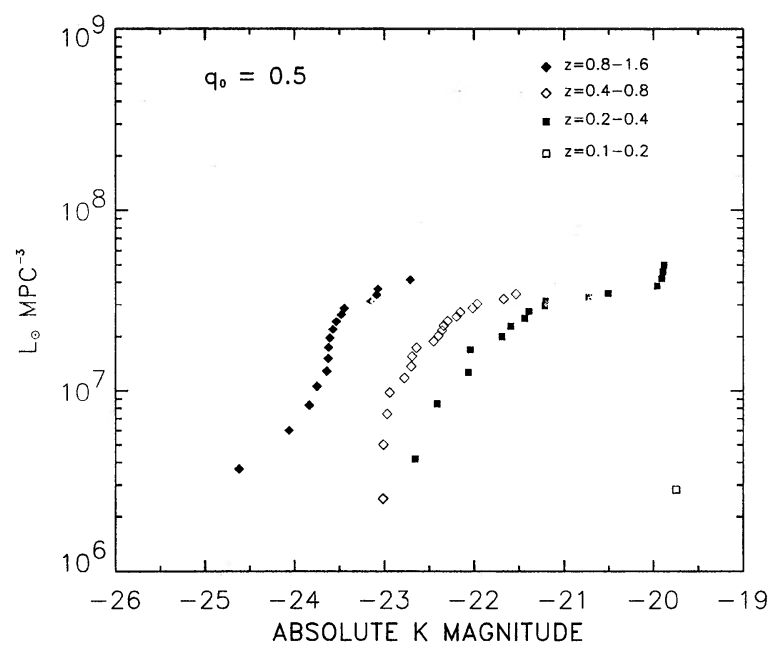

FIG. 24. Rest frame $K$-band light density of blue galaxies as a function of redshift interval for a $K \leqslant 20.5$ sample. The smooth increase in the luminosity of the most luminous forming galaxies with increasing redshift is readily apparent.

$L_{\odot} \mathrm{Mpc}^{-3}$ at the higher redshift interval and $2.4 \times 10^{8} L_{\odot}$ $\mathrm{Mpc}^{-3}$ at the lower.

Figure 24 shows the cumulative $K$-band light density for the blue objects over a wider range of redshifts where we now use a $K=20.5$ sample, since to this magnitude all objects with $U K(A B)<1.3$ are contained in the combined $K, I$, and $B$ sample. Figure 24 shows the smooth increase in luminosity of the most luminous forming galaxies with increasing redshift. It is this smooth change of the maximum "forming" luminosity with redshift which, when combined with the distance modulus and the rest-frame blue colors of the "forming"' galaxies, results in a near caustic where the apparent magnitude at which the forming galaxies enter the samples is almost the same at all redshifts-roughly $B \geqslant 23$ or $K \geqslant 20$. This is illustrated at a more detailed level in Fig. 25 where we show the "forming" galaxies selected either by UV color or [O II] equivalent width (filled squares) in $z-B$ space superimposed over all $B<24.5$ galaxies (pluses). The first "forming" galaxies to enter the sample lie at around $B \sim 22$ and $z \sim 0.3$, and it is this minimum which resulted in the "small blue galaxies" being the first detected population of forming galaxies (Cowie et al. 1991; Glazebrook et al. 1995a), but beyond $B=23$ forming galaxies enter at essentially all redshifts to at least $z=1.7$ (Fig. 25). It is the rather remarkable cancellation of the "forming" $M_{K}$ and the distance modulus which results in the excess in the $B$-band counts at $B \gtrsim 23$ and the rapid blueing which sets in at the faint $B$ and $K$ magnitudes.

As was shown in Sec. 3.3 the $K$ luminosity density rises with increasing redshift at intermediate redshifts but then begins to drop at higher redshift $(z \geqq 0.6)$. This effect can be easily understood if the $M_{K}-t_{d}-z$ diagrams of the previous subsection are interpreted as showing the formation of present-day luminous galaxies (exponential star-forming galaxies) since in this case much of the present-day $K$ luminosity density, which, as we have shown above, arises primarily 

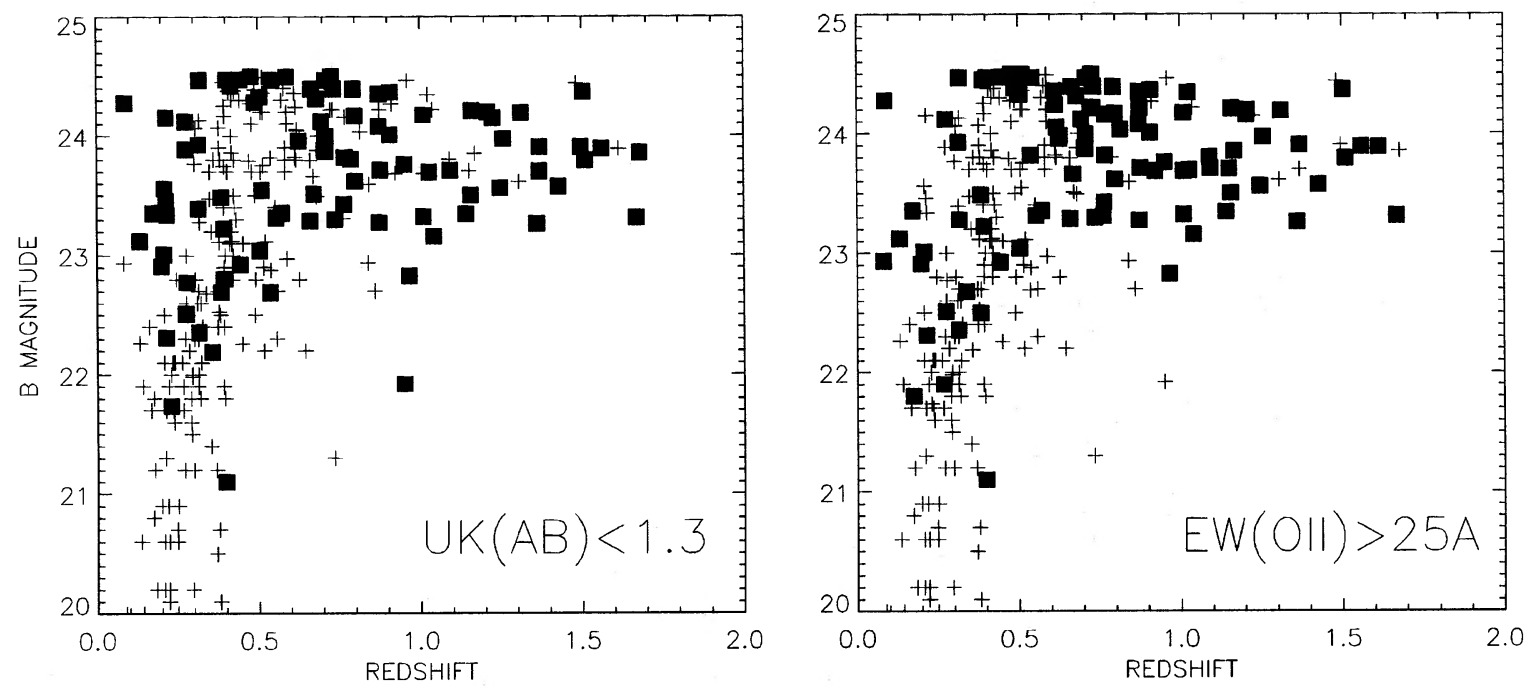

FIG. 25. $B$ magnitude vs redshift for all $B \leqslant 24.5$ galaxies (pluses) in the present sample or in Songaila et al. (1994) compared to "forming" galaxies (filled squares) chosen either on the basis of rest-frame UV-IR color (left panel) or rest-frame [O II] equivalent width (right panel). The forming galaxies first enter at $B \sim 22, z \sim 0.3$ and then spread to a wide range of redshifts at fainter magnitudes. This coincidence is responsible for the simultaneous rise in the $B$ counts at $B \geqslant 23$ and the rapid blueing which sets in at faint $B$ and $K$ magnitudes.

at $M_{K}<-23+5 \log _{10} h_{50}$, was being assembled at the earlier redshifts. The $K$ luminosity density peaks when the more massive galaxies cease to form, and only then begins to passively decline. By contrast, if the rapid formers were bursts in smaller objects, the bulk of the $K$ light would already be present in invariant objects at these redshifts and we would expect to see a smooth increase in $\lambda_{K}$ with increasing $z$ from the passive evolution.

\subsection{The Universal Rate of Star Formation}

The $K$ light density discussed in the previous subsection is an integral measure of the accumulated light density as a function of redshift and so its evolution provides a history of universal star formation. An alternative approach is to look at the rest-frame ultraviolet light density, which measures the rate of massive star formation and so, for an invariant stellar mass function, the rate of star formation. The ultraviolet light density should therefore roughly measure the rate of accumulation of the light density which, when convolved through the transfer function from stellar evolution, would predict the $K$ light density evolution. It therefore provides an independent check of the results of the previous subsection.

Cowie (1988) first suggested that a very model-invariant way to describe this was by comparing the extragalactic background surface brightness at these frequencies with the production of metals, since both relate to the same massive stars and so avoid the uncertainties in the stellar mass function. Songaila et al. (1990) give this relation as

$$
\begin{aligned}
& S_{\nu}=3.6 \times 10^{-25} \\
& \times\left(\frac{\rho Z}{10^{-34} \mathrm{~g} \mathrm{~cm}^{-3}}\right) \operatorname{ergs~} \mathrm{cm}^{-2} \mathrm{~s}^{-1} \mathrm{~Hz}^{-1} \mathrm{deg}^{-2},
\end{aligned}
$$

where $S_{\nu}$ is the present sky surface brightness at the restframe ultraviolet frequency $\nu$ and $\rho Z$ is the present mass density of metals in the universe. This equation is independent of the cosmological parameters. For comparison purposes we shall assume a local $\rho Z=(2 \rightarrow 6) \times 10^{-34} h_{50}^{2}$ gm $\mathrm{cm}^{-3}$ following the various estimates given in Cowie (1988) and Songaila et al. (1990).

Figure 26 shows $S_{\nu}$ for objects satisfying $U K(A B)<1.3$ divided by redshift bin compared with the expected sky surface brightness which should be produced by all galaxy formation, the range of which is shown by the dotted lines. The upper right panel shows the rest-frame ultraviolet surface brightness of all galaxies in the samples irrespective of their colors, while the lower right panel shows that of only those objects whose rest-frame ultraviolet-infrared colors imply rapid formation. Finally, in the two left-hand panels (solid lines) we show the extragalactic background light (EBL) for the rapidly forming galaxies of the lower right panel, cut at an absolute $3500 \AA$ magnitude of $-20.5+5 \log _{10} h_{50}$. From equation (4) of Cowie (1988), this roughly corresponds to an $\dot{M}$ of $30 M_{\odot} \mathrm{yr}^{-1}$ for a Salpeter IMF, and so is approximately the luminosity which will form a $10^{11} M_{\odot}$ galaxy in $3 \times 10^{9} \mathrm{yr}$. The two left-hand panels again illustrate the point that larger galaxies form earlier than smaller ones, but also illustrate the second point, that we are seeing a large fraction $(10 \%-20 \%)$ of the light needed to make all observed galaxies in the rapid formers. In fact, this conclusion is substantially understated by the solid lines because of internal extinction. If we allow for one magnitude of internal extinction in the galaxies, the solid line of massive forming galaxies in the upper left panel moves to the dashed line and essentially all of galaxy formation can be accounted for.

The right-hand lower panel shows the total observed light in all forming galaxies, again assuming no extinction, and in 

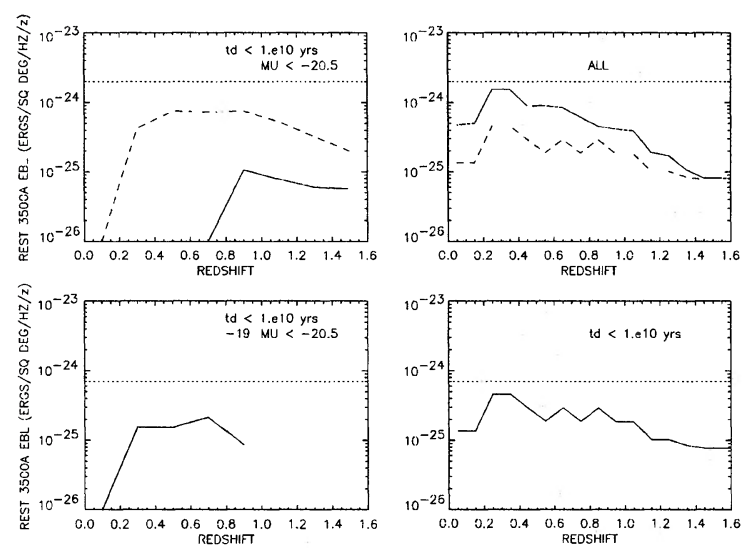

FIG. 26. Galaxy contributions to the ultraviolet sky surface brightness as a function of redshift, rest-frame galaxy color, and absolute rest $U$ magnitude. The dotted lines show the range of values expected from the present-day metallicity. The left two panels show the contributions to the extragalactic background light (EBL) from forming galaxies $\left(t_{d}<10^{10}\right.$ years) over two intervals for absolute rest $U$ magnitude. A comparison of the solid curves for the left two panels shows again that large galaxies form earlier than small ones. The dashed line in the upper left panel shows the effect of correcting for one magnitude of internal extinction on the contribution of observed galaxies to the EBL. It is apparent that we are already seeing a large fraction of the light required to make all observed galaxies. The right two panels compare the contributions from all galaxies and "forming" galaxies to the EBL, assuming no extinction. In the upper right panel the solid line shows the contribution from all galaxies compared to that of the "forming" galaxies (dashed line), which are also shown in the lower right panel. Again, these curves show that we are observing the bulk of galaxy formation, with roughly half of the star formation occurring in rapidly forming galaxies (upper right panel).

the upper right we compare this with the light of all observed galaxies. Again we see that, with even a small amount of extinction and any level of incompleteness at the high- $z$ end, we are seeing the bulk of galaxy formation. The upper panel also makes the point that roughly half of the star formation occurs in the fast-forming galaxies, while the other half occurs as ongoing star formation in older galaxies, as would be expected in exponential star-forming models, but not in burst models.

\section{CONCLUSIONS}

In this paper we have presented evidence that some substantial fraction of all galaxy formation occurred between $z=0.8$ and $z=1.6$, and also that galaxy formation took place in "downsizing," with more massive galaxies forming at higher redshift. The late galaxy formation accounts for the rapid evolution in galaxy colors, while the differential evolution-with the most massive galaxies forming earliest-results in relatively little evolution in $K_{*}$ and $B_{*}$ between $z=0$ and $z=1$ and so results in the galaxy redshift distribution being well described by no-evolution models at $z<1$.

The present data fit well with recent studies of the evolution of the intergalactic gas. In particular, it now appears that the $\Omega$ in damped Ly $\alpha$ absorption systems seen in quasar spectra was relatively constant from $z=2$ to 5 , with a value of around $2.5 \times 10^{-3} h_{50}$, very similar to that in present-day galaxies (Sec. 6.2), but drops rapidly at $z<2$ (StorrieLombardi et al. 1995) presumably as a consequence of the galaxy formation discussed here. The onset of rapid galaxy formation at these redshifts also allows us to understand the low metallicity in most $z \gtrsim 2$ damped Ly $\alpha$ systems (Pettini et al. 1994) and the correspondingly required cosmic chemical evolution between $z=2$ and $z=0$ (Pei \& Fall 1995).

The "downsizing"' is a more remarkable result. It allows a very natural interpretation of the data at the expense of a remarkably regular fall of the maximum luminosity of "forming" galaxies with redshift. It is possible to envisage theoretical models which utilize feedback through the intergalactic gas to achieve this type of result as, for example, if the ongoing galaxy formation increased the IGM pressure and so reduced the characteristic "forming" mass, but it remains to be seen whether a fully self-consistent description can be constructed which also fits the quasar absorption line data.

We would like to thank Karl Glazebrook, Stéphane Charlot, Tom Shanks, Caryl Gronwall, Tom Broadhurst, and most particularly the referee, Richard Ellis, for their many helpful comments on an earlier version of this paper. We are grateful to Tom Bida, Peter Gillingham, Joel Aycock, Teresa Chelminiak, Barabara Schaefer, and Wayne Wack for their extensive help in obtaining the observations with the Keck LRIS spectrograph. This work was partly based on observations obtained with the NASA/ESA Hubble Space Telescope and was supported by the State of Hawaii and by NASA through grants number GO-5399.01-93A, GO-5922.01-94A, and GO-6626.01-95A from the Space Telescope Science Institute, which is operated by AURA, Inc., under NASA contract NAS5-26555.

\section{REFERENCES}

Broadhurst, T. J., Ellis, R. S., \& Glazebrook, K. 1992, Nature, 355, 55 Broadhurst, T. J., Ellis, R. S., \& Shanks, T. 1988, MNRAS, 235, 827

Bruzual A., G., \& Charlot, S. 1993, ApJ, 405, 538

Carlberg, R. G. 1992, ApJ, 399, L31

Coleman, G. D., Wu, C.-C., \& Weedman, D. W. 1980, ApJS, 43, 393

Colless, M. M., Ellis, R. S., Taylor, K., \& Hook, R. N. 1990, MNRAS, 244, 408

Colless, M. M., Ellis, R. S., Broadhurst, T. J., Taylor, K., \& Peterson, B. A. 1993, MNRAS, 261, 19
Cowie, L. L. 1988, in The Post-Recombination Universe, edited by N. Kaiser and A. Lazenby (Kluwer, Dordrecht), p. 1

Cowie, L. L., Gardner, J. P., Hu, E. M., Songaila, A., Hodapp, K.-W., \& Wainscoat, R. J., 1994, ApJ, 434, 114 (Paper I)

Cowie, L. L., Hu, E. M., \& Songaila, A. 1995a, Nature, 377, 603

Cowie, L. L., Hu, E. M., \& Songaila, A. 1995b, AJ, 110, 1576

Cowie, L. L., Songaila, A., \& Hu, E. M. 1991, Nature, 354, 460

Driver, S. P., Windhorst, R. A., Ostrander, E. J., Keel, W. C., Griffiths, R. E., \& Ratnatunga, K. U. 1995, ApJ, 449, L23 
Eales, S. 1993, ApJ, 404, 51

Ellis, R. S., Colless, M., Broadhurst, T., Heyl, J., \& Glazebrook, K. 1996 MNRAS, 280, 235

Gallagher, J. S., Bushouse, H., \& Hunter, D. A. 1989, AJ, 97, 700

Glazebrook, K., Ellis, R. S., Colless, M. M., Broadhurst, T. J., Allington-

Smith, J. R., \& Tanvir, N. R. 1995a, MNRAS, 273, 157 (GECBAT)

Glazebrook, K., Peacock, J. A., Miller, L., \& Collins, C. A., 1995b, MNRAS, 266, 65

Gronwall, C., \& Koo, D. C. 1995, ApJ, 440, L1

Kennicutt, R. C., Jr. 1992, ApJ, 388, 310

King, C. R., \& Ellis, R. S. 1985, ApJ, 288, 456

LeFèvre, O., Crampton, D., Hammer, F., Lilly, S. J., \& Tresse, L. 1994, ApJ, 423, L89

Lilly, S. J., Hammer, F. LeFèvre, O., \& Crampton, D. 1995a, ApJ, 455, 75 Lilly, S. J., Tresse, L., Hammer, F., Crampton, D., \& LeFèvre, O. 1995b, ApJ, 455, 108
Loveday, J., Peterson, B. A., Efstathiou, G., \& Maddox, S. J. 1992, ApJ, 390,338

Mobasher, B., Ellis, R. S., \& Sharples, R. M. 1986, MNRAS, 223, 11

Mobasher, B., Sharples, R. M., \& Ellis, R. S. 1993, MNRAS, 263, 560

Oke, J. B., et al., 1995, PASP, 107, 375

Pei, Y., \& Fall, M. 1995. ApJ, 454, 69

Pettini, M., Smith, L. J., Hunstead, R. W., \& King, D. L. 1994, ApJ, 426, 79

Rocca-Volmerange, B., \& Guiderdoni, B. 1990, MNRAS, 247, 166

Shanks, T. 1990, in The Galactic and Extragalactic Background Radiation, IAU Symposium No. 139, edited by S. Bowyer and C. Leinert (Kluwer, Dordrecht), p. 269

Songaila, A., Cowie, L. L., Hu, E. M., \& Gardner, J. P. 1994, ApJS, 94, 461 Songaila, A., Cowie, L. L., \& Lilly, S. J. 1990, ApJ, 348, 371

Steidel, C. C., Dickinson, M., \& Persson, S. E. 1994, ApJ, 437, L75

Storrie-Lombardi, L. J., McMahon, R. J., Irwin, M. J., \& Hazard, C. 1995, in QSO Absorption Lines, edited by G. Meylan (Springer, Berlin), p. 47 